\title{
Boundary layer expansions for initial value problems with two complex time variables
}

\section{A. Lastra ${ }^{1 *}$ (D) and S. Malek}

"Correspondence:

alberto.lastra@uah.es

'Departamento de Física y

Matemáticas, University of Alcalá

Alcalá de Henares, Spain

Full list of author information is

available at the end of the article

\section{Springer}

\begin{abstract}
We study a family of partial differential equations in the complex domain, under the action of a complex perturbation parameter $\epsilon$. We construct inner and outer solutions of the problem and relate them to asymptotic representations via Gevrey asymptotic expansions with respect to $\epsilon$ in adequate domains. The asymptotic representation leans on the cohomological approach determined by the Ramis-Sibuya theorem.
\end{abstract}

MSC: $35 \mathrm{C} 10 ; 35 \mathrm{C} 20$

Keywords: Asymptotic expansion; Borel-Laplace transform; Fourier transform; Initial value problem; Formal power series; Boundary layer; Singular perturbation

\section{Introduction}

The main aim in this work is to describe the analytic solutions and asymptotic behavior of the solutions of a family of initial value problems in the complex domain. Such a family consists of partial differential equations in two complex time variables of the form

$$
Q\left(\partial_{z}\right) u\left(t_{1}, t_{2}, z, \epsilon\right)=P\left(t_{1}^{k_{1}+1} \partial_{t_{1}}, t_{2}^{k_{2}+1} \partial_{t_{2}}, \partial_{z}, z, \epsilon\right) u\left(t_{1}, t_{2}, z, \epsilon\right)+f\left(t_{1}, t_{2}, z, \epsilon\right),
$$

under given initial data $u\left(0, t_{2}, z, \epsilon\right) \equiv u\left(t_{1}, 0, z, \epsilon\right) \equiv 0$. Here, $Q(X) \in \mathbb{C}[X]$ and $P\left(T_{1}, T_{2}, Z\right.$, $z, \epsilon)$ stands for a polynomial in $\left(T_{1}, T_{2}, Z\right)$ with holomorphic coefficients w.r.t. $(z, \epsilon)$ on $H_{\beta} \times D\left(0, \epsilon_{0}\right)$, where $H_{\beta}$ stands for the horizontal strip in the complex plane

$$
H_{\beta}:=\{z \in \mathbb{C}:|\operatorname{Im}(z)|<\beta\}
$$

for some $\beta>0$, and $D\left(0, \epsilon_{0}\right) \subseteq \mathbb{C}$ stands for the open disc centered at the origin with radius $\epsilon_{0}$ for some small $\epsilon_{0}>0$. The symbol $\epsilon$ acts as a small complex perturbation parameter in the equation. Moreover, $k_{1}, k_{2}$ are positive integers with $1 \leq k_{1}<k_{2}$. The forcing term, constructed in detail in Sect. 2, turns out to be a holomorphic function in $\mathbb{C}^{2} \times H_{\beta^{\prime}} \times$ $D\left(0, \epsilon_{0}\right)$. In this paper, we also adopt the notation $\bar{D}(0, r)$ for the closed disc centered at $0 \in \mathbb{C}$ and radius $r>0$.

The precise constrains involving the parameters involved in each of the equations determining the family of PDEs under study are described in Sect. 2.

(c) The Author(s) 2020. This article is licensed under a Creative Commons Attribution 4.0 International License, which permits use sharing, adaptation, distribution and reproduction in any medium or format, as long as you give appropriate credit to the original author(s) and the source, provide a link to the Creative Commons licence, and indicate if changes were made. The images or other third party material in this article are included in the article's Creative Commons licence, unless indicated otherwise in a credit line to the material. If material is not included in the article's Creative Commons licence and your intended use is not permitted by statutory regulation or exceeds the permitted use, you will need to obtain permission directly from the copyright holder. To view a copy of this licence, visit http://creativecommons.org/licenses/by/4.0/. 
This is the continuation of a series of works devoted to the study of PDEs in the complex domain under the action of two complex time variables. In [18], the authors studied a family of nonlinear initial value Cauchy problems of the form

$$
\begin{aligned}
Q\left(\partial_{z}\right) \partial_{t_{1}} \partial_{t_{2}} u\left(t_{1}, t_{2}, z, \epsilon\right)= & \left(P_{1}\left(\partial_{z}, \epsilon\right) u\left(t_{1}, t_{2}, z, \epsilon\right)\right)\left(P_{2}\left(\partial_{z}, \epsilon\right) u\left(t_{1}, t_{2}, z, \epsilon\right)\right) \\
& +P\left(t_{1}, t_{2}, \partial_{t_{1}}, \partial_{t_{2}}, \partial_{z}, \epsilon\right) u\left(t_{1}, t_{2}, z, \epsilon\right)+f\left(t_{1}, t_{2}, z, \epsilon\right),
\end{aligned}
$$

where the terms in $Q, P, P_{1}, P_{2}$ are such that the action of $t_{1}$ and $t_{2}$ is symmetric. Moreover, we assume that the polynomial

$$
\mathcal{P}\left(t_{1}, t_{2}, \partial_{t_{1}}, \partial_{t_{2}}, \partial_{z}, \epsilon\right):=Q\left(\partial_{z}\right) \partial_{t_{1}} \partial_{t_{2}}-L_{1}\left(t_{1}, t_{2}, \partial_{t_{1}}, \partial_{t_{2}}, \partial_{z}, \epsilon\right)
$$

where $L_{1}$ involves leading terms of the differential operator $P$, can be factorized in such a way that each of the factors only depends on one of the time variables, i.e.,

$$
\mathcal{P}\left(t_{1}, t_{2}, \partial_{t_{1}}, \partial_{t_{2}}, \partial_{z}, \epsilon\right)=\mathcal{P}_{1}\left(t_{1}, \partial_{t_{1}}, \partial_{z}, \epsilon\right) \mathcal{P}_{2}\left(t_{2}, \partial_{t_{2}}, \partial_{z}, \epsilon\right)
$$

From this symmetric configuration one is able to construct families of analytic bounded solutions $u_{d_{h}}\left(t_{1}, t_{2}, z, \epsilon\right) \in \mathcal{O}_{b}\left(\mathcal{T}_{1} \times \mathcal{T}_{2} \times H_{\beta^{\prime \prime}} \times \mathcal{E}_{h}\right)$ for every $0 \leq h \leq \iota-1$, where $\mathcal{T}_{1}, \mathcal{T}_{2}, \mathcal{E}_{j}$ stand for open bounded sectors with vertex at the origin in $\mathbb{C}, \beta^{\prime \prime}>0$, and $\left(\mathcal{E}_{h}\right)_{0 \leq h \leq l-1}$ is a good covering of $\mathbb{C}^{\star}$ (see Definition 3). Moreover, an asymptotic behavior of such solutions can be observed with respect to the perturbation parameter $\epsilon$. Indeed, there exists a formal power series $\epsilon \mapsto \hat{u}\left(t_{1}, t_{2}, z, \epsilon\right) \in \mathbb{E} \llbracket \epsilon \rrbracket$, where $\mathbb{E}$ stands for the Banach space of holomorphic and bounded functions defined in $\mathcal{T}_{1} \times \mathcal{T}_{2} \times H_{\beta^{\prime \prime}}$ with the norm of the supremum, which turns out to be a formal solution of (2). In addition to this, a multisummability result joins both analytic and formal solutions (see [18], Theorem 2).

In the second study [15], the property of symmetry of the equations drops, and $\mathcal{P}$ in (3) is no longer factorizable into two terms which only present dependence on one of the time variables. This asymmetry causes that the procedure followed in [18] is no longer valid in that second framework and the procedure followed differs from that in [18].

In both studies, a Borel-Laplace method is applied. In the symmetric case, the analytic solution is constructed as the Laplace transform with respect to $\tau_{1}$ and $\tau_{2}$ of an auxiliary function $\omega\left(\tau_{1}, \tau_{2}\right)$, which is well defined in a domain of the form $\left(S_{1} \cup D(0, \rho)\right) \times\left(S_{2} \cup\right.$ $D(0, \rho))$ for some $\rho>0$ and certain sectors $S_{1}, S_{2}$ with vertex at the origin. Moreover, such a function admits an exponential growth at infinity with respect to $\tau_{1} \in S_{1}$ and $\tau_{2} \in S_{2}$. This is the suitable configuration in order to apply Borel-Laplace techniques on each of the variables involved and achieve summability results (see Sect. 5.1). On the other hand, the asymmetric settings in the problem considered in [15] cause the function $\omega\left(\tau_{1}, \tau_{2}\right)$ only be defined in sets of the form $S_{1} \times\left(S_{2} \cup D(0, \rho)\right)$, and a small divisor phenomenon is observed. Therefore, the summability conditions are not satisfied, and a different approach, focused on studying the natural domains and asymptotic behavior of $\omega\left(\tau_{1}, \tau_{2}\right)$, and applying summability results asymmetrically, has to be followed.

In this sense, this work is concerned with a family of equations in which none of the previous strategies is satisfactory. On the one hand, the symmetric situation does not hold in the present work, so the strategy followed in [18] is not available. On the other hand, 
the strategy considered in [15] does not apply because the auxiliary function $\omega\left(\tau_{1}, \tau_{2}\right)$ requires that, at least for one of the variables, a neighborhood of the origin is contained in its domain of definition. This is not the case, so a summability procedure cannot be followed. The reason for failure is that the deformation path accomplished when computing the difference of two consecutive solutions of the main problem, written as the Laplace transform $\omega$, is no longer applicable. A small divisor phenomenon occurs, which does not allow to determine the Gevrey orders involved in the relationship between the analytic and the formal solution. The precise reasoning on the failure of this procedure is detailed in Sect. 2.1.

A second novelty in the present work is the appearance of two different kinds of families of analytic solutions of the main problem for which one can give a picture of their asymptotic behavior with respect to the perturbation parameter. Following the terminology in the study of boundary layer solutions of equations, we distinguish the inner solutions (see Sect. 4.1) and the outer solutions (see Sect. 4.2) of the main problem and describe their asymptotic representation with respect to the perturbation parameter near the origin. A recent work by the authors [17] constructs boundary layer expansions for certain initial value problem with merging turning points, regarding inner and outer solutions, which only considers the action of one time variable in the equation. In that previous work and also in the present work, the Gevrey orders of the asymptotic representation of the inner and outer solutions are different in general. As mentioned, we observe a comparable phenomenon in the present situation. However, in our context the inner solutions might not be $\lambda_{1} k_{1}$-summable in general for some $\lambda_{1}>0$ to be precised.

The so-called inner and outer expansions are of great interest in mathematics under the theory of matched asymptotic expansions. For a detailed theory on this subject, we refer to classical textbooks such as $[3,7,11,20-22]$. For the general aspects on Gevrey asymptotic expansions in this context, we refer to the book [9].

It is worth mentioning that the special solution $u\left(t_{1}, t_{2}, z, \epsilon\right)$ of the main problem under study (1), which is constructed in this work, satisfies the partial differential equation

$$
\tilde{P}_{p, q}(u):=\left\{\left(\frac{\epsilon^{\lambda_{1} k_{1}}}{k_{1}} t_{1}^{k_{1}+1} \partial_{t_{1}}\right)^{p}-\left(\frac{\epsilon^{\lambda_{2} k_{2}}}{k_{2}} t_{2}^{k_{2}+1} \partial_{t_{2}}\right)^{q}\right\} u=0
$$

for $(p, q) \in \mathbb{N}^{2}$ satisfying $p k_{1}=q k_{2}$. Moreover, the forcing term $f\left(t_{1}, t_{2}, z, \epsilon\right)$ in (1) satisfies

$$
\tilde{P}_{p, q} f=0 .
$$

One may choose $(p, q):=\left(k_{2}, k_{1}\right)$ or other generator $(p, q)=\left(\operatorname{gcd}\left(k_{1}, k_{2}\right)\right)^{-1}\left(k_{2}, k_{1}\right)$. Therefore, the present study can be seen from the point of view of the asymptotic behavior of a family of special solutions to the system of singularly perturbed partial differential equations (1) and (4) satisfying the compatibility condition (5).

The study of singularly perturbed PDEs in the complex domain is a topic of increasing interest. In 2015, Yamazawa and Yoshino [23] studied parametric Borel summability in semilinear systems of PDEs of fuchsian type and of combined irregular and fuchsian type by Yoshino [24].

The theory of monomial summability was put forward by Canalis-Durand, MozoFernández, and Schäfke in [4]. Recently, Carrillo and Mozo-Fernández have studied fur- 
ther properties on monomial summability and Borel-Laplace methods on this theory in $[5,6]$, and this technique has been successfully applied to families of singularly perturbed ODEs and PDEs.

We now give a general overview of the sections in which the present study is divided and the main results obtained. The statement of the main problem under consideration is settled in Sect. 2, where we give arguments on the reason of failure of the methods used in $[15,18]$ in this family of PDEs (see Sect. 2.1) and determine the shape of the analytic solution as a Laplace-like transform of a function related to the meromorphic kernel $\Omega$ provided in (18) (see Sect. 2.2): given two good coverings of $\mathbb{C}^{\star},\left(\mathcal{E}_{h_{1}}^{0}\right)_{0 \leq h_{1} \leq l_{1}-1}$ and $\left(\mathcal{E}_{h_{2}}^{\infty}\right)_{0 \leq h_{2} \leq t_{2}-1}$, with the first good covering consisting of sectors with wide enough opening (see Definition 3), we construct two sets of analytic solutions of (1) in the form

$$
\begin{aligned}
u_{\xi_{h_{j}}}\left(t_{1}, t_{2}, z, \epsilon\right):= & \frac{1}{(2 \pi)^{1 / 2}} \int_{-\infty}^{\infty} \int_{L_{d_{h_{j}}}} \omega(u, m, \epsilon) \exp \left(-\left(\frac{u}{\epsilon^{\lambda_{1}} t_{1}}\right)^{k_{1}}-\left(\frac{u}{\epsilon^{\lambda_{2}} t_{2}}\right)^{k_{2}}\right) \\
& \times \exp (i z m) \frac{d u}{u} d m, \quad j=1,2 .
\end{aligned}
$$

The elements of the first family are constructed on a domain of the form $\mathcal{T}_{1} \times\left(\mathcal{T}_{2} \cap\right.$ $\left.D\left(0, \rho_{2}\right)\right) \times H_{\beta^{\prime}} \times \mathcal{E}_{h_{1}}^{0}$ for some $\rho_{2}>0$. The elements in the second family are constructed on domains of the form $\mathcal{T}_{1} \times \mathcal{T}_{2, \epsilon} \times H_{\beta^{\prime}} \times \mathcal{E}_{h_{2}}^{\infty}$. Here, $\mathcal{T}_{1}$ is a bounded sector, $\mathcal{T}_{2}$ is an unbounded sector, and the direction $\xi_{h} \in \mathbb{R}$ is an appropriate argument, the set $\mathcal{T}_{2, \epsilon}$ is a bounded sector that depends on $\epsilon \in \mathcal{E}_{h_{2}}^{\infty}$ and tends to infinity with $\epsilon \rightarrow 0 ; \lambda_{1}, \lambda_{2} \in \mathbb{N}$. The function $\omega(u, m, \epsilon)$ comes as a result of a fixed point argument in the Borel plane, in certain Banach spaces of functions (see Sect. 2.3 and Sect. 2.4). We finally relate the analytic solutions to an asymptotic representation in different subdomains achieving the construction and asymptotic results on the inner solutions (see Sect. 4.1) and on the outer solutions (see Sect. 4.2). In both situations, we provide differences of consecutive solutions (in the sense that they are associated with consecutive sectors in the fixed good covering) and apply the Ramis-Sibuya theorem (see Theorem (RS)) to arrive at the existence of a common asymptotic representation of all the inner solutions and an asymptotic representation of all the outer solutions. This asymptotic behavior appears in the form of Gevrey asymptotic expansions of order $1 /\left(\lambda_{1} k_{1}\right)$ with respect to the perturbation parameter $\epsilon$ on $\mathcal{E}_{h_{2}}^{\infty}$ regarding the inner solutions, whilst $\lambda_{2} k_{2}$-Gevrey summability can be observed regarding the outer solutions, with respect to the perturbation parameter (see Theorem 4).

The paper is organized as follows.

In Sect. 2, we state the main problem under study and analyze different ways to approach the problem. The section ends with the construction of the solution to an auxiliary problem in the Borel plane within a Banach space of functions with exponential growth and decay. In Sect. 3, we study the solution of an auxiliary problem, which turns out to be crucial in the sequel. Section 4 is devoted to the construction of the analytic solution of the main problem in addition to the inner and outer solutions. The work ends in Sect. 5 with the study of the parametric Gevrey asymptotic expansions of both types of solutions in appropriate domains, with respect to the perturbation parameter. The last section is focused on the technical proof of Lemma 4, left at the end of the paper for the sake of clarity in the ongoing argumentation. 


\section{Statement of the main problem and solution of an auxiliary problem}

Our main aim in this work is to provide analytic and formal solutions to the main problem under study (1) and give information about the asymptotic behavior relating both. In this section, we detail the elements involved in the main problem under study and provide different approaches which might be followed in order to search analytic and asymptotically related formal solutions.

Let $1 \leq k_{1}<k_{2}$ and $D_{1}, D_{2} \geq 2$ be integers. We also fix $\lambda_{1}, \lambda_{2} \in \mathbb{N}$. For $1 \leq \ell_{1} \leq D_{1}$ and $1 \leq \ell_{2} \leq D_{2}$, we consider nonnegative integers $\delta_{\ell_{1}}, \delta_{\ell_{2}}$, and $\Delta_{\ell_{1} \ell_{2}}$.

We assume that

$$
\begin{aligned}
& \Delta_{D_{1} D_{2}}=\lambda_{1} k_{1} \delta_{D_{1}}+\lambda_{2} k_{2} \delta_{D_{2}}, \quad \lambda_{2} k_{2}>\lambda_{1} k_{1}, \\
& \Delta_{\ell_{1} \ell_{2}}>\lambda_{1} k_{1} \delta_{\ell_{1}}+\lambda_{2} k_{2} \delta_{\ell_{2}}, \quad k_{1} \delta_{D_{1}}+k_{2} \delta_{D_{2}} \geq k_{1} \delta_{\ell_{1}}+k_{2} \delta_{\ell_{2}}, \\
& 1 \leq \ell_{1} \leq D_{1}-1,1 \leq \ell_{2} \leq D_{2}-1 .
\end{aligned}
$$

We consider polynomials with complex coefficients $Q, R_{D_{1} D_{2}}$, and $R_{\ell_{1} \ell_{2}}$ for every $1 \leq$ $\ell_{1} \leq D_{1}-1$ and $1 \leq \ell_{2} \leq D_{2}-1$. We assume that

$$
\frac{Q(i m)}{R_{D_{1} D_{2}}(i m)} \in A_{Q, R_{D_{1} D_{2}}}, \quad m \in \mathbb{R}
$$

where $A_{Q, R_{D_{1}}, R_{D_{2}}}$ stands for the sectorial annulus $A_{Q, R_{D_{1} D_{2}}}$ defined by

$$
\left\{z \in \mathbb{C}: r_{Q, R_{D_{1} D_{2}}}^{1} \leq|z| \leq r_{Q, R_{D_{1} D_{2}}}^{2}, \arg (z) \in\left(\alpha_{Q, R_{D_{1} D_{2}}}, \beta_{Q, R_{D_{1} D_{2}}}\right)\right\}
$$

for some $0<r_{Q, R_{D_{1} D_{2}}}^{1}<r_{Q, R_{D_{1} D_{2}}}^{2}$ and $\alpha_{Q, R_{D_{1} D_{2}}}, \beta_{Q, R_{D_{1} D_{2}}} \in \mathbb{R}$, with $\alpha_{Q, R_{D_{1} D_{2}}}<\beta_{Q, R_{D_{1} D_{2}}}$. In addition to that, we assume

$$
\begin{aligned}
& \operatorname{deg}\left(R_{\ell_{1} \ell_{2}}\right) \leq \operatorname{deg}\left(R_{D_{1} D_{2}}\right), \quad 1 \leq \ell_{1} \leq D_{1}-1,1 \leq \ell_{2} \leq D_{2}-1, \\
& R_{D_{1} D_{2}}(i m) \neq 0, \quad m \in \mathbb{R} .
\end{aligned}
$$

We consider the main initial value problem under study:

$$
\begin{aligned}
Q\left(\partial_{z}\right) u\left(t_{1}, t_{2}, z, \epsilon\right) & \\
= & \epsilon^{\Delta D_{1} D_{2}}\left(t_{1}^{k_{1}+1} \partial_{t_{1}}\right)^{\delta_{D_{1}}}\left(t_{2}^{k_{2}+1} \partial_{t_{2}}\right)^{\delta_{D_{2}}} R_{D_{1} D_{2}}\left(\partial_{z}\right) u\left(t_{1}, t_{2}, z, \epsilon\right) \\
& +\sum_{\substack{1 \leq \ell_{1} \leq D_{1}-1 \\
1 \leq \ell_{2} \leq D_{2}-1}} \epsilon^{\Delta \ell_{1} \ell_{2}}\left(t_{1}^{k_{1}+1} \partial_{t_{1}}\right)^{\delta_{\ell_{1}}}\left(t_{2}^{k_{2}+1} \partial_{t_{2}}\right)^{\delta_{\ell_{2}}} c_{\ell_{1} \ell_{2}}(z, \epsilon) R_{\ell_{1} \ell_{2}}\left(\partial_{z}\right) u\left(t_{1}, t_{2}, z, \epsilon\right) \\
& +f\left(t_{1}, t_{2}, z, \epsilon\right),
\end{aligned}
$$

under given initial conditions $u\left(0, t_{2}, z, \epsilon\right) \equiv 0$ and $u\left(t_{1}, 0, z, \epsilon\right) \equiv 0$.

Let $\epsilon_{0}>0$. For every $0 \leq \ell_{1} \leq D_{1}-1$ and $0 \leq \ell_{2} \leq D_{2}-1$, the functions $c_{\ell_{1} \ell_{2}}(z, \epsilon)$ are holomorphic on $H_{\beta} \times D\left(0, \epsilon_{0}\right)$. They are defined by the inverse Fourier transform

$$
c_{\ell_{1} \ell_{2}}(z, \epsilon):=\mathcal{F}^{-1}\left(m \mapsto c_{\ell_{1} \ell_{2}}(m, \epsilon)\right)(z)=\frac{1}{(2 \pi)^{1 / 2}} \int_{-\infty}^{\infty} C_{\ell_{1} \ell_{2}}(m, \epsilon) e^{i z m} d m
$$


where $m \mapsto C_{\ell_{1} \ell_{2}}(m, \epsilon)$ is continuous for $m \in \mathbb{R}$ and satisfies uniform bounds with respect to $\epsilon \in D\left(0, \epsilon_{0}\right)$. More precisely, there exists $\mathcal{C}_{\ell_{1} \ell_{2}}>0$ such that

$$
\sup _{\epsilon \in D\left(0, \epsilon_{0}\right)}\left|C_{\ell_{1} \ell_{2}}(m, \epsilon)\right| \leq \frac{\mathcal{C}_{\ell_{1} \ell_{2}}}{(1+|m|)^{\mu}} \exp (-\beta|m|), \quad m \in \mathbb{R}
$$

for some $\mu>0$.

$f$ is a holomorphic function in $\mathbb{C}^{\star} \times \mathbb{C} \times H_{\beta^{\prime}} \times\left(D\left(0, \epsilon_{0}\right) \backslash\{0\}\right)$ for every $0<\beta^{\prime}<\beta$. The details of its construction are given in Sect. 2.2.

We look for time rescaled solutions of (10) of the specific form

$$
u\left(t_{1}, t_{2}, z, \epsilon\right)=\mathcal{F}^{-1}\left(m \mapsto U\left(\epsilon^{\lambda_{1}} t_{1}, \epsilon^{\lambda_{2}} t_{2}, m, \epsilon\right)\right)(z) .
$$

Accordingly, we assume that the forcing term writes in the same manner:

$$
f\left(t_{1}, t_{2}, z, \epsilon\right)=\mathcal{F}^{-1}\left(m \mapsto F\left(\epsilon^{\lambda_{1}} t_{1}, \epsilon^{\lambda_{2}} t_{2}, m, \epsilon\right)\right)(z),
$$

where $F\left(T_{1}, T_{2}, m, \epsilon\right)$ is holomorphic with respect to $\left(T_{1}, T_{2}\right)$ on certain domain in $\mathbb{C}^{2}$, continuous with respect to $m$ in $\mathbb{R}$, and holomorphic with respect to $\epsilon$ on the disc $D\left(0, \epsilon_{0}\right)$.

In view of (6), the classical properties of Fourier inverse transform, and the definition of $f$ and $c_{\ell_{1} \ell_{2}}$, we get that the expression $U\left(T_{1}, T_{2}, m, \epsilon\right)$ turns out to be a solution of

$$
\begin{aligned}
Q(i m) & U\left(T_{1}, T_{2}, m, \epsilon\right) \\
= & \left(T_{1}^{k_{1}+1} \partial_{T_{1}}\right)^{\delta_{D_{1}}}\left(T_{2}^{k_{2}+1} \partial_{T_{2}}\right)^{\delta_{D_{2}}} R_{D_{1} D_{2}}(i m) U\left(T_{1}, T_{2}, m, \epsilon\right) \\
& +\sum_{\substack{1 \leq \ell_{1} \leq D_{1}-1 \\
1 \leq \ell_{2} \leq D_{2}-1}} \epsilon^{\Delta_{\ell_{1} \ell_{2}}-\lambda_{1} k_{1} \delta_{\ell_{1}}-\lambda_{2} k_{2} \delta_{\ell_{2}}}\left(T_{1}^{k_{1}+1} \partial_{T_{1}}\right)^{\delta_{\ell_{1}}}\left(T_{2}^{k_{2}+1} \partial_{T_{2}}\right)^{\delta_{\ell_{2}}} \\
& \times \frac{1}{(2 \pi)^{1 / 2}} \int_{-\infty}^{\infty} C_{\ell_{1} \ell_{2}}\left(m-m_{1}, \epsilon\right) R_{\ell_{1} \ell_{2}}\left(i m_{1}\right) U\left(T_{1}, T_{2}, m_{1}, \epsilon\right) d m_{1} \\
& +F\left(T_{1}, T_{2}, m, \epsilon\right) .
\end{aligned}
$$

\subsection{A first approach}

As a first approach, one is tempted to follow the techniques used in the previous works of the authors dealing with singularly perturbed partial differential equations in two complex time variables such as [12-14]. On the one hand, the family of equations studied in [18] shows a symmetric role of the time variables in the equation. Although this is the case for (10), in that previous study it holds that the principal part of any of the equations in the family is factorizable as a product of two operators which split the dependence on the time variables. For this reason, that procedure is no longer valid in the present framework. On the other hand, the study made in [15] does not fit the main problem under study, as it can be deduced from the forthcoming argument.

We search for solutions $U\left(T_{1}, T_{2}, m, \epsilon\right)$ of (14) of the form of a double Laplace transform

$$
\begin{aligned}
& U_{d_{1}, d_{2}}\left(T_{1}, T_{2}, m, \epsilon\right) \\
& \quad=\frac{k_{1} k_{2}}{(2 \pi)^{1 / 2}} \int_{L_{d_{1}}} \int_{L_{d_{2}}} \omega\left(u_{1}, u_{2}, m, \epsilon\right) \exp \left(-\left(\frac{u_{1}}{T_{1}}\right)^{k_{1}}-\left(\frac{u_{2}}{T_{2}}\right)^{k_{2}}\right) \frac{d u_{1}}{u_{1}} \frac{d u_{2}}{u_{2}}
\end{aligned}
$$


along well chosen halflines $L_{d_{j}}=\mathbb{R}_{+} e^{d_{j} \sqrt{-1}}$, with $d_{j} \in \mathbb{R}, j=1,2$. Accordingly, we assume that the forcing term $F\left(T_{1}, T_{2}, m, \epsilon\right)$ is written in a similar way:

$$
F\left(T_{1}, T_{2}, m, \epsilon\right)=\frac{k_{1} k_{2}}{(2 \pi)^{1 / 2}} \int_{L_{d_{1}}} \int_{L_{d_{2}}} \tilde{F}\left(u_{1}, u_{2}, m, \epsilon\right) \exp \left(-\left(\frac{u_{1}}{T_{1}}\right)^{k_{1}}-\left(\frac{u_{2}}{T_{2}}\right)^{k_{2}}\right) \frac{d u_{1}}{u_{1}} \frac{d u_{2}}{u_{2}},
$$

where $\tilde{F}\left(u_{1}, u_{2}, m, \epsilon\right)$ defines a holomorphic function on $\mathbb{C}^{2}$ with respect to $\left(u_{1}, u_{2}\right)$, continuous with respect to $m \in \mathbb{R}$, holomorphic with respect to $\epsilon$ on $D\left(0, \epsilon_{0}\right)$.

Let

$$
P_{m}\left(\tau_{1}, \tau_{2}\right):=Q(i m)-\left(k_{1} \tau_{1}^{k_{1}}\right)^{\delta_{D_{1}}}\left(k_{2} \tau_{2}^{k_{2}}\right)^{\delta_{D_{2}}} R_{D_{1} D_{2}}(i m) .
$$

We consider the related problem

$$
\begin{aligned}
& \left(Q(i m)-\left(k_{1} \tau_{1}^{k_{1}}\right)^{\delta_{D_{1}}}\left(k_{2} \tau_{2}^{k_{2}}\right)^{\delta_{D_{2}}} R_{D_{1} D_{2}}(i m)\right) \omega\left(\tau_{1}, \tau_{2}, m, \epsilon\right) \\
& =\sum_{\substack{1 \leq \ell_{1} \leq D_{1}-1 \\
1 \leq \ell_{2} \leq D_{2}-1}} \epsilon^{\Delta \ell_{1} \ell_{2}-\lambda_{1} k_{1} \delta_{\ell_{1}}-\lambda_{2} k_{2} \delta \ell_{2}}\left(k_{1} \tau_{1}^{k_{1}}\right)^{\delta \ell_{1}}\left(k_{2} \tau_{2}^{k_{2}}\right)^{\delta \ell_{2}} \\
& \times \frac{1}{(2 \pi)^{1 / 2}} \int_{-\infty}^{\infty} C_{\ell_{1} \ell_{2}}\left(m-m_{1}, \epsilon\right) R_{\ell_{1} \ell_{2}}\left(i m_{1}\right) \omega\left(\tau_{1}, \tau_{2}, m_{1}, \epsilon\right) d m_{1} \\
& +\tilde{F}\left(\tau_{1}, \tau_{2}, m, \epsilon\right) .
\end{aligned}
$$

Under the previous construction, the following proposition holds.

Proposition 1 Under the previous construction leading to (17), it holds that the possible actual holomorphic solutions $\omega\left(\tau_{1}, \tau_{2}, m, \epsilon\right)$ of $(17)$ cannot be defined on any set of the form $\left(S_{1} \cup D(0, \rho)\right) \times S_{2}$ (resp. $\left.S_{1} \times\left(S_{2} \cup D(0, \rho)\right)\right)$ with respect to $\left(\tau_{1}, \tau_{2}\right)$ for any $\rho>0$ and any unbounded sectors $S_{1}, S_{2}$ with vertex at the origin in $\mathbb{C}$, i.e., $S_{j}=\left\{z \in \mathbb{C}: \arg (z) \in\left(\alpha_{j}, \beta_{j}\right)\right\}$ for some real numbers $\alpha_{j}<\beta_{j}$ and $j=1,2$.

Proof Since equation (17) exhibits a symmetric behavior with respect to $\tau_{1}$ and $\tau_{2}$, we only give details on the first of the previous statements, whilst the second follows from a symmetric argument.

Fix $m \in \mathbb{R}$ and let $\rho_{0}>0$. We consider $\tau_{2} \in S_{2}$ such that

$$
\left|\tau_{2}\right| \geq\left(\frac{r_{Q, R_{D_{1} D_{2}}}^{2}}{\left(\rho_{0} / 2\right)^{k_{1} \delta_{D_{1}}}} \frac{1}{k_{1}^{\delta_{D_{1}}} k_{2}^{\delta_{D_{2}}}}\right)^{\frac{1}{k_{2} \delta_{2}}}
$$

We derive that any $\tau_{1} \in \mathbb{C}$ such that $P_{m}\left(\tau_{1}, \tau_{2}\right)=0$ would satisfy that

$$
\tau_{1}^{k_{1} \delta_{D_{1}}} \tau_{2}^{k_{2} \delta_{D_{2}}}=\frac{Q(i m)}{R_{D_{1} D_{2}}(i m)} \frac{1}{k_{1}^{\delta_{D_{1}}} k_{2}^{\delta_{D_{2}}}},
$$

which entails

$$
\left|\tau_{1}\right|^{k_{1} \delta_{D_{1}}} \leq \frac{1}{\left|\tau_{2}\right|^{k_{2} \delta_{D_{2}}}} r_{Q, R_{D_{1} D_{2}}}^{2} \frac{1}{k_{1}^{\delta_{D_{1}}} k_{2}^{\delta_{D_{2}}}} \leq\left(\frac{\rho_{0}}{2}\right)^{k_{1} \delta_{D_{1}}} .
$$


It follows that all the $k_{1} \delta_{D_{1}}$ roots of $\tau_{1} \mapsto P_{m}\left(\tau_{1}, \tau_{2}\right)$ for such choice of $\tau_{2}$ belong to the disc $D\left(0, \rho_{0}\right)$. The limit $\rho_{0} \rightarrow 0$ concludes the result.

As a matter of fact, a small divisor phenomenon is observed, which does not allow a summability procedure. Moreover, the possible actual holomorphic solutions $\omega\left(\tau_{1}, \tau_{2}, m, \epsilon\right)$ of (17) are only expected to be well defined and holomorphic on products of sectors with infinite radius, say $S_{1} \times S_{2}$. This construction does not allow us to use the procedure applied neither in [18] nor [15] in order to analyze the asymptotic properties of the solutions with respect to the small perturbation parameter $\epsilon$ (see the introduction of this work for further details).

\subsection{Second approach}

In view of the failure of the approaches in $[15,18]$ (see Sect. 2.1), we need to adopt another perspective.

We search for solutions of (14) of the special form

$$
\begin{aligned}
U_{d}\left(T_{1}, T_{2}, m, \epsilon\right) & =\int_{L_{d}} \omega(u, m, \epsilon) \exp \left(-\left(\frac{u}{T_{1}}\right)^{k_{1}}-\left(\frac{u}{T_{2}}\right)^{k_{2}}\right) \frac{d u}{u} \\
& =\int_{L_{d}} \omega(u, m, \epsilon) \Omega\left(u, T_{1}, T_{2}\right) \frac{d u}{u}
\end{aligned}
$$

where $L_{d}$ stands for a halfline departing from the origin and with bisecting direction of argument given by $d \in \mathbb{R}$ for some $d \in \mathbb{R}$ to be determined. Accordingly, we assume that the forcing term $F\left(T_{1}, T_{2}, m, \epsilon\right)$ is expressed in a similar manner. Let $\psi: \mathbb{C} \times \mathbb{R} \times D\left(0, \epsilon_{0}\right) \rightarrow$ $\mathbb{C}$ be an entire function with respect to the first variable, continuous in $\mathbb{R}$ with respect to the second one, and holomorphic with respect to the third variable on the disc $D\left(0, \epsilon_{0}\right)$. Moreover, there exist $C_{\psi}, \beta, \mu, v \in \mathbb{R}$, with $C_{\psi}, \beta, v>0$ and

$$
\mu>1+\operatorname{deg}\left(R_{\ell_{1} \ell_{2}}\right), \quad 1 \leq \ell_{1} \leq D_{1}-1,1 \leq \ell_{2} \leq D_{2}-1,
$$

such that the following bound holds:

$$
|\psi(\tau, m, \epsilon)| \leq \frac{C_{\psi}}{(1+|m|)^{\mu}} e^{-\beta|m|} \exp \left(v|\tau|^{k^{\prime}}\right)|\tau|
$$

for all $(\tau, m, \epsilon) \in \mathbb{C} \times \mathbb{R} \times D\left(0, \epsilon_{0}\right)$ and some $k_{1}<k^{\prime}<k_{2}$. In this situation, it is straightforward to check that the function

$$
F\left(T_{1}, T_{2}, m, \epsilon\right):=\int_{L_{\gamma}} \psi(u, m, \epsilon) \exp \left(-\left(\frac{u}{T_{1}}\right)^{k_{1}}-\left(\frac{u}{T_{2}}\right)^{k_{2}}\right) \frac{d u}{u},
$$

where $L_{\gamma}=[0, \infty) e^{\gamma \sqrt{-1}}$, can spin around the origin in order to guarantee that $F$ is a holomorphic function on $\mathbb{C}^{\star} \times \mathbb{C}$ with respect to $\left(T_{1}, T_{2}\right)$ by analytic continuation. The corresponding forcing term $f\left(t_{1}, t_{2}, m, \epsilon\right)$ expressed in (13) is holomorphic on $\mathbb{C}^{\star} \times \mathbb{C} \times H_{\beta^{\prime}} \times$ $\left(D\left(0, \epsilon_{0}\right) \backslash\{0\}\right)$ for all $0<\beta^{\prime}<\beta$.

Our idea consists on merging the double integral Laplace transform along the product $L_{d_{1}} \times L_{d_{2}}$ from the first approach in (15) into a simple integral along a halfline $L_{d} \subseteq \mathbb{C}$. Geometrically, it consists in a projection on the diagonal part $(u, u) \in \mathbb{C}^{2}, u \in \mathbb{C}$ of the space $\mathbb{C}^{2}$. The advantage is that the related problem associated with the Borel map $w(u, m, \epsilon)$ 
(see (21)) involves now $P_{m}(\tau, \tau)$ as a denominator which is this time well defined on a full neighborhood of 0 w.r.t. $\tau$ and analytically continuable along unbounded sectors $S_{d}$ with suitable directions $d \in \mathbb{R}$. As a result, the asymptotic analysis in $\epsilon$ becomes a tractable task. The drawback of this approach concerns the class of equations we are able to handle, which is reduced compared to our previous studies and contains linear PDEs with special time reliance.

Remark Observe that in the case $k_{1}=k_{2}$, the function $U_{d}$ turns out to be a Laplace transform of order $k_{1}$ in the meromorphic function

$$
\Omega\left(T_{1}, T_{2}\right)=\frac{1}{\left(\frac{1}{T_{1}}\right)^{k_{1}}+\left(\frac{1}{T_{2}}\right)^{k_{1}}},
$$

near $0 \in \mathbb{C}^{2}$. That situation is directly linked to a summability procedure with respect to a germ of function in $\mathbb{C}^{2}$, as described in [19]. However, in our situation, the function $\Omega$ is meromorphic near 0 , not analytic.

Under the hypothesis that the solution of (14) is of the form (18), $\omega$ in (18) solves the problem

$$
\begin{aligned}
& \left(Q(i m)-\left(k_{1} \tau^{k_{1}}\right)^{\delta_{D_{1}}}\left(k_{2} \tau^{k_{2}}\right)^{\delta_{D_{2}}} R_{D_{1} D_{2}}(i m)\right) \omega(\tau, m, \epsilon) \\
& =\sum_{\substack{1 \leq \ell_{1} \leq D_{1}-1 \\
1 \leq \ell_{2} \leq D_{2}-1}} \epsilon^{\Delta \ell_{1} \ell_{2}-\lambda_{1} k_{1} \delta_{\ell_{1}}-\lambda_{2} k_{2} \delta_{\ell_{2}}}\left(k_{1} \tau^{k_{1}}\right)^{\delta \ell_{1}}\left(k_{2} \tau^{k 2}\right)^{\delta \ell_{2}} \\
& \quad \times \frac{1}{(2 \pi)^{1 / 2}} \int_{-\infty}^{\infty} C_{\ell_{1} \ell_{2}}\left(m-m_{1}, \epsilon\right) R_{\ell_{1} \ell_{2}}\left(i m_{1}\right) \omega\left(\tau, m_{1}, \epsilon\right) d m_{1}+\psi(\tau, m, \epsilon) .
\end{aligned}
$$

We substitute (17) by (21) as an auxiliary problem in order to solve the main equation. In the next section, we study some spaces of functions which are involved in the construction of the solution of (21).

\subsection{Banach spaces of functions with exponential growth and decay}

The Banach spaces described in this section are modified versions of those appearing in [12]. We omit the details which can be derived directly from that work and the variations of that norm stated in $[15,18]$.

We fix positive real numbers $\beta, \mu$, v, with $\mu>1$, and an integer $k^{\prime}>0$. Let $\rho>0$ and $S_{d}$ be an open and unbounded sector with bisecting direction $d \in \mathbb{R}$, i.e.,

$$
S_{d}=\{z \in \mathbb{C}: \arg (z) \in(d-a, d+a)\}
$$

for some $a>0$. We denote by $\bar{D}(0, \rho)$ the closed disc centered at 0 and positive radius $\rho$.

Firstly, we recall some classical properties of the inverse Fourier transform, which are used in our construction.

Definition 1 We denote by $E_{(\beta, \mu)}$ the vector space of continuous functions $h: \mathbb{R} \rightarrow \mathbb{C}$ such that

$$
\|h(m)\|_{(\beta, \mu)}=\sup _{m \in \mathbb{R}}(1+|m|)^{\mu} \exp (\beta|m|)|h(m)|
$$

is finite. The space $E_{(\beta, \mu)}$ equipped with the norm $\|\cdot\|_{(\beta, \mu)}$ is a Banach space. 
Proposition 2 Let $f \in E_{(\beta, \mu)}$. The inverse Fourier transform of $f$, given by

$$
\mathcal{F}^{-1}(f)(x)=\frac{1}{(2 \pi)^{1 / 2}} \int_{-\infty}^{+\infty} f(m) \exp (i x m) d m, \quad x \in \mathbb{R},
$$

can be extended to an analytic function on the horizontal strip

$$
H_{\beta}=\{z \in \mathbb{C} /|\operatorname{Im}(z)|<\beta\} .
$$

Let $\phi(m):=\operatorname{imf}(m) \in E_{(\beta, \mu-1)}$. Then the following statements hold:

(a) $\partial_{z} \mathcal{F}^{-1}(f)(z)=\mathcal{F}^{-1}(\phi)(z)$ for $z \in H_{\beta}$.

(b) Let $g \in E_{(\beta, \mu)}$, and consider the convolution product of $f$ and $g$ :

$$
\psi(m):=\frac{1}{(2 \pi)^{1 / 2}} \int_{-\infty}^{\infty} f\left(m-m_{1}\right) g\left(m_{1}\right) d m_{1} .
$$

Then $\psi \in E_{(\beta, \mu)}$ and $\mathcal{F}^{-1}(f)(z) \mathcal{F}^{-1}(g)(z)=\mathcal{F}^{-1}(\psi)(z), z \in H_{\beta}$.

Definition 2 We write $\operatorname{Exp}_{\left(\nu, \beta, \mu, k^{\prime}\right)}^{d}$ for the vector space of continuous complex-valued functions $(\tau, m) \mapsto h(\tau, m)$, defined on $\left(S_{d} \cup \bar{D}(0, \rho)\right) \times \mathbb{R}$, holomorphic with respect to $\tau$ on $S_{d} \cup D(0, \rho)$ such that

$$
\|h(\tau, m)\|_{\left(\nu, \beta, \mu, k^{\prime}\right)}:=\sup _{\tau \in S_{d} \cup \bar{D}(0, \rho), m \in \mathbb{R}}(1+|m|)^{\mu} e^{\beta|m|} \exp \left(-\nu|\tau|^{k^{\prime}}\right) \frac{1}{|\tau|}|h(\tau, m)|
$$

is finite. The normed space $\left(\operatorname{Exp}_{\left(v, \beta, \mu, k^{\prime}\right)}^{d}\|\cdot\|_{\left(v, \beta, \mu, k^{\prime}\right)}\right)$ is a Banach space.

The next result is straighforward from the definition of the norm $\|\cdot\|_{\left(v, \beta, \mu, k^{\prime}\right)}$.

Lemma 1 Let $(\tau, m) \mapsto a(\tau, m)$ be a bounded continuous function of $\left(\bar{D}(0, \rho) \cup S_{d}\right) \times \mathbb{R}$, holomorphic with respect to $\tau$ on $D(0, \rho) \cup S_{d}$. Then, for every $h \in \operatorname{Exp}_{\left(\nu, \beta, \mu, k^{\prime}\right)}^{d}$, it holds that $a(\tau, m) h(\tau, m) \in \operatorname{Exp}_{\left(v, \beta, \mu, k^{\prime}\right)}^{d}$ and

$$
\|a(\tau, m) h(\tau, m)\|_{\left(\nu, \beta, \mu, k^{\prime}\right)} \leq\left(_{(\tau, m) \in\left(\bar{D}(0, \rho) \cup S_{d}\right) \times \mathbb{R}}|a(\tau, m)|\right)\|h(\tau, m)\|_{\left(\nu, \beta, \mu, k^{\prime}\right)} .
$$

The proof of the next result follows analogous arguments as those in Proposition 2 in [16], and we refer to that work for a complete proof.

Proposition 3 Let $R_{1}, R_{2} \in \mathbb{C}[X]$ such that

$$
\operatorname{deg}\left(R_{1}\right) \geq \operatorname{deg}\left(R_{2}\right), \quad R_{1}(i m) \neq 0, \quad \mu>\operatorname{deg}\left(R_{2}\right)+1 .
$$

Given $f \in E_{(\beta, \mu)}$ and $g \in \operatorname{Exp}_{\left(\nu, \beta, \mu, k^{\prime}\right)}^{d}$, then it holds that the function

$$
\Phi(\tau, m):=\frac{1}{R_{1}(i m)} \int_{-\infty}^{\infty} f\left(m-m_{1}\right) R_{2}\left(i m_{1}\right) g\left(\tau, m_{1}\right) d m_{1}
$$

is an element of $\operatorname{Exp}_{\left(v, \beta, \mu, k^{\prime}\right)}^{d}$, and there exists $C_{1}>0$ such that

$$
\|\Phi(\tau, m)\|_{\left(\nu, \beta, \mu, k^{\prime}\right)} \leq C_{1}\|f(m)\|_{(\beta, \mu)}\|g(\tau, m)\|_{\left(\nu, \beta, \mu, k^{\prime}\right)} .
$$




\subsection{Solution of an auxiliary equation}

At this point, we provide a brief summary on the strategy to trace. We continue with the approach described in Sect. 2.2, searching for solutions of (14) in the form (18). In this section, we guarantee the existence of $\omega(\tau, m, \epsilon)$ by means of a fixed point argument in the Banach space of functions introduced in Sect. 2.3.

At this point, we follow a similar guideline as the one initiated in our former study [12]. We consider the following polynomial:

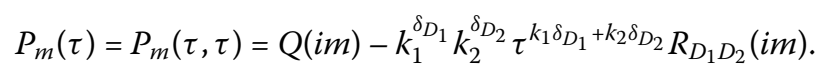

In the following, we need lower bounds of the expression $P_{m}(\tau)$ with respect to $m$ and $\tau$. In order to achieve this goal, we can factorize the polynomial w.r.t. $\tau$, namely

$$
P_{m}(\tau)=-k_{1}^{\delta_{D_{1}}} k_{2}^{\delta_{D_{2}}} R_{D_{1} D_{2}}(i m) \prod_{l=0}^{k_{1} \delta_{D_{1}}+k_{2} \delta_{D_{2}}-1}\left(\tau-q_{l}(m)\right),
$$

where its roots $q_{l}(m)$ can be displayed explicitly as follows:

$$
\begin{aligned}
q_{l}(m)= & \left(\frac{|Q(i m)|}{\left|R_{D_{1} D_{2}}(i m)\right| k_{1}^{\delta_{D_{1}}} k_{2}^{\delta_{D_{2}}}}\right)^{\frac{1}{k_{1} \delta_{D_{1}}+k_{2} \delta_{D_{2}}}} \\
& \times \exp \left(\frac{\sqrt{-1}}{k_{1} \delta_{D_{1}}+k_{2} \delta_{D_{2}}}\left(\arg \left(\frac{Q(i m)}{R_{D_{1} D_{2}}(i m) k_{1}^{\delta_{D_{1}}} k_{2}^{\delta_{D_{2}}}}\right)+2 \pi l\right)\right)
\end{aligned}
$$

for all $0 \leq l \leq k_{1} \delta_{D_{1}}+k_{2} \delta_{D_{2}}-1$, for all $m \in \mathbb{R}$.

We set an unbounded sector $S_{d}$ centered at 0 , a small disc $D(0, \rho)$, and we adjust the sector $A_{Q, R_{D_{1} D_{2}}}$ in a way that the following condition holds: a constant $\mathfrak{m}>0$ can be chosen with

$$
\left|\tau-q_{l}(m)\right| \geq \mathfrak{m}(1+|\tau|)
$$

for all $0 \leq l \leq k_{1} \delta_{D_{1}}+k_{2} \delta_{D_{2}}-1$, all $m \in \mathbb{R}$, provided that $\tau \in S_{d} \cup D(0, \rho)$. Indeed, the inclusion (8) implies in particular that all the roots $q_{l}(m), 0 \leq l \leq k_{1} \delta_{D_{1}}+k_{2} \delta_{D_{2}}-1$, remain apart of some neighborhood of the origin, i.e., satisfy $\left|q_{l}(m)\right| \geq 2 \rho$ for an appropriate choice of $\rho>0$. Furthermore, when the opening of $A_{Q, R_{D_{1} D_{2}}}$ is taken close enough to 0 , all these roots $q_{l}(m)$ stay inside a union $\mathcal{U}$ of unbounded sectors centered at 0 that do not cover a full neighborhood of 0 in $\mathbb{C}^{*}$. We assign a sector $S_{d}$ with

$$
S_{d} \cap \mathcal{U}=\emptyset .
$$

By construction, the quotients $q_{l}(m) / \tau$ fall apart some small disc centered at 1 in $\mathbb{C}$ for all $\tau \in S_{d}, m \in \mathbb{R}, 0 \leq l \leq k_{1} \delta_{D_{1}}+k_{2} \delta_{D_{2}}-1$. Then (25) follows.

We are now ready to supply lower bounds for $P_{m}(\tau)$. 
Lemma 2 A constant $C_{P}>0$ (depending on $k_{1}, k_{2}, \delta_{D_{1}}, \delta_{D_{2}}, \mathfrak{m}$ ) can be found with

$$
\left|P_{m}(\tau)\right| \geq C_{P}\left|R_{D_{1} D_{2}}(i m)\right|(1+|\tau|)^{k_{1} \delta_{D_{1}}+k_{2} \delta_{D_{2}}}
$$

for all $\tau \in S_{d} \cup D(0, \rho)$, all $m \in \mathbb{R}$.

Proof Departing from factorization (24), the lower bounds (25) entail

$$
\left|P_{m}(\tau)\right| \geq k_{1}^{\delta_{D_{1}}} k_{2}^{\delta_{D_{2}}} \mathfrak{m}^{k_{1} \delta_{D_{1}}+k_{2} \delta_{D_{2}}}\left|R_{D_{1} D_{2}}(\mathrm{im})\right|(1+|\tau|)^{k_{1} \delta_{D_{1}}+k_{2} \delta_{D_{2}}}
$$

for all $\tau \in S_{d} \cup D(0, \rho)$.

Lemma 3 Assume that conditions (6)-(9) hold on the elements involved in problem (10), with forcing term $f$ determined by the construction and conditions in (19)-(20). We single out a sector $S_{d}$ that fulfills the constraints from the construction above.

Then there exist $\epsilon_{0}, \varpi>0$ and $\xi_{\psi}>0$ (depending on $k_{1}, k_{2}, \delta_{D_{1}}, \delta_{D_{2}}, Q, R_{D_{1} D_{2}}$ ) such that if $C_{\psi} \leq \xi_{\psi}$, then for every $\epsilon \in D\left(0, \epsilon_{0}\right)$ the map $\mathcal{H}_{\epsilon}$ defined by

$$
\begin{aligned}
\mathcal{H}_{\epsilon}(\omega(\tau, m)):= & \frac{1}{P_{m}(\tau)}\left(\sum_{\substack{1 \leq \ell_{1} \leq D_{1}-1 \\
1 \leq \ell_{2} \leq D_{2}-1}} \epsilon^{\Delta \ell_{1} \ell_{2}-\lambda_{1} k_{1} \delta \ell_{1}-\lambda_{2} k_{2} \delta \ell_{2}}\left(k_{1} \tau^{k}\right)^{\ell_{\ell_{1}}}\left(k_{2} \tau^{k_{2}}\right)^{\delta \ell_{2}}\right. \\
& \left.\times \frac{1}{(2 \pi)^{1 / 2}} \int_{-\infty}^{\infty} C_{\ell_{1} \ell_{2}}\left(m-m_{1}, \epsilon\right) R_{\ell_{1} \ell_{2}}\left(i m_{1}\right) \omega\left(\tau, m_{1}\right) d m_{1}\right) \\
& +\frac{1}{P_{m}(\tau)} \psi(\tau, m, \epsilon)
\end{aligned}
$$

satisfies the following properties:

(i) $\mathcal{H}_{\epsilon}(\bar{B}(0, \varpi)) \subseteq B(0, \varpi)$, where $\bar{B}(0, \varpi)$ is the closed disc of radius $\varpi>0$, in $\operatorname{Exp}_{\left(v, \beta, \mu, k^{\prime}\right)}^{d}$.

(ii) We have

$$
\begin{aligned}
& \|\| \mathcal{H}_{\epsilon}\left(\omega_{1}\right)-\mathcal{H}_{\epsilon}\left(\omega_{2}\right)\left\|_{\left(\nu, \beta, \mu, k^{\prime}\right)} \leq \frac{1}{2}\right\| \omega_{1}-\omega_{2} \|_{\left(\nu, \beta, \mu, k^{\prime}\right)} \\
& \text { for all } \omega_{1}, \omega_{2} \in \bar{B}(0, \varpi) \subseteq \operatorname{Exp}_{\left(v, \beta, \mu, k^{\prime}\right)}^{d} .
\end{aligned}
$$

Proof Take $\omega \in \operatorname{Exp}_{\left(v, \beta, \mu, k^{\prime}\right)}^{d}$ and $\epsilon \in D\left(0, \epsilon_{0}\right)$. Let $1 \leq \ell_{1} \leq D_{1}-1$ and $1 \leq \ell_{2} \leq D_{2}-1$. Bearing in mind Lemma 1, Proposition 3, from (9), (11), and (26), we have

$$
\begin{aligned}
& \| \epsilon^{\Delta \ell_{1} \ell_{2}-\lambda_{1} k_{1} \delta_{\ell_{1}}-\lambda_{2} k_{2} \delta_{\ell_{2}}}\left(k_{1} \tau^{k_{1}}\right)^{\delta_{\ell_{1}}}\left(k_{2} \tau^{k_{2}}\right)^{\delta_{\ell_{2}}} \\
& \times \frac{1}{(2 \pi)^{1 / 2}} \int_{-\infty}^{\infty} \frac{C_{\ell_{1} \ell_{2}}\left(m-m_{1}, \epsilon\right) R_{\ell_{1} \ell_{2}}\left(i m_{1}\right)}{P_{m}(\tau)} \omega\left(\tau, m_{1}\right) d m_{1} \|_{\left(v, \beta, \mu, k^{\prime}\right)} \\
& \leq C\left(\epsilon_{0}\right) \sup _{\tau \in S_{d} \cup \bar{D}(0, \rho), m \in \mathbb{R}} \frac{|\tau|^{k_{1} \delta_{\ell_{1}}+k_{2} \delta_{\ell_{2}}}}{(1+|\tau|)^{k_{1} \delta_{D_{1}}+k_{2} \delta_{D_{2}}}} \\
& \times\left\|\frac{1}{R_{D_{1} D_{2}}(i m)} \int_{-\infty}^{\infty} C_{\ell_{1} \ell_{2}}\left(m-m_{1}, \epsilon\right) R_{\ell_{1} \ell_{2}}(i m) \omega\left(\tau, m_{1}\right) d m_{1}\right\|_{\left(v, \beta, \mu, k^{\prime}\right)} \\
& \leq C\left(\epsilon_{0}\right) C_{1} C_{2} \mathcal{C}_{\ell_{1} \ell_{2}}\|\omega(\tau, \epsilon)\|_{\left(\nu, \beta, \mu, k^{\prime}\right)} \leq C\left(\epsilon_{0}\right) C_{1} C_{2} \mathcal{C}_{\ell_{1} \ell_{2}} \varpi,
\end{aligned}
$$


where

$$
C\left(\epsilon_{0}\right)=\sup _{\substack{1 \leq \ell_{1} D_{1}-1 \\ 1 \leq \ell_{2} \leq D_{2}-1}} \frac{\epsilon_{0}^{\Delta_{\ell_{1} \ell_{2}}-\lambda_{1} k_{1} \delta_{\ell_{1}}-\lambda_{2} k_{2} \delta_{\ell_{2}}} k_{1}^{\delta_{\ell_{1}}-\delta_{D_{1}}} k_{2}^{\delta_{\ell_{2}}-\delta_{D_{2}}}}{(2 \pi)^{1 / 2} C_{P} \mathfrak{m}^{k_{1} \delta_{D_{1}}+k_{2} \delta_{D_{2}}}}>0,
$$

is such that $C\left(\epsilon_{0}\right) \rightarrow 0$ for $\epsilon_{0} \rightarrow 0$, and

$$
C_{2}=\sup _{\tau \in S_{d} \cup \bar{D}(0, \rho), m \in \mathbb{R}} \frac{|\tau|^{k_{1} \delta_{\ell_{1}}+k_{2} \delta_{\ell_{2}}}}{(1+|\tau|)^{k_{1} \delta_{D_{1}}+k_{2} \delta_{D_{2}}}} .
$$

On the other hand, in view of (9), (19), and (26), we derive that

$$
\left\|\frac{1}{P_{m}(\tau)} \psi(\tau, m, \epsilon)\right\|_{\left(\nu, \beta, \mu, k^{\prime}\right)} \leq \sup _{\substack{\tau \in S_{d} \cup \bar{D}(0, \rho) \\ m \in \mathbb{R}}} \frac{C_{\psi}}{C_{p}\left|R_{D_{1} D_{2}}(i m)\right|(1+|\tau|)^{k_{1} \delta_{D_{1}}+k_{2} \delta_{D_{2}}}} \leq C_{3} \xi_{\psi}
$$

for some $C_{3}>0$.

Let $\varpi, \xi_{\psi}, \epsilon_{0}>0$ such that $C\left(\epsilon_{0}\right) C_{1} C_{2}\left(\sum_{\substack{1 \leq \ell_{1} \leq D_{1}-1 \\ 1 \leq \ell_{2} \leq D_{2}-1}} \mathcal{C}_{\ell_{1} \ell_{2}}\right) \varpi+\xi_{\psi} C_{3} \leq \varpi$. Under this choice, we get that $\mathcal{H}_{\epsilon}$ is such that $\mathcal{H}_{\epsilon}(\bar{B}(0, \varpi)) \subseteq \bar{B}(0, \varpi)$. For the second part of the proof, we choose $\omega_{1}, \omega_{2} \in \operatorname{Exp}_{\left(v, \beta, \mu, k^{\prime}\right)}^{d}$, with $\left\|\omega_{j}\right\|_{\left(\nu, \beta, \mu, k^{\prime}\right)} \leq \varpi$. Analogous estimates as in (28) yield

$$
\begin{aligned}
& \left\|\epsilon^{\Delta \ell_{1} \ell_{2}-\lambda_{1} k_{1} \delta_{\ell_{1}}-\lambda_{2} k_{2} \delta_{\ell_{2}}}\left(k_{1} \tau^{k_{1}}\right)^{\delta_{\ell_{1}}}\left(k_{2} \tau^{k_{2}}\right)^{\delta_{\ell_{2}}} \frac{R_{\ell_{1} \ell_{2}}(i m)}{P_{m}(\tau)}\left(\omega_{1}(\tau, m)-\omega_{2}(\tau, m)\right)\right\|_{\left(v, \beta, \mu, k^{\prime}\right)} \\
& \quad \leq C\left(\epsilon_{0}\right) C_{1} C_{2} \mathcal{C}_{\ell_{1} \ell_{2}}\left\|\omega_{1}(\tau, m)-\omega_{2}(\tau, m)\right\|_{\left(v, \beta, \mu, k^{\prime}\right)}
\end{aligned}
$$

and consequently

$$
\begin{aligned}
\left\|\mathcal{H}_{\epsilon}\left(\omega_{1}\right)-\mathcal{H}_{\epsilon}\left(\omega_{2}\right)\right\|_{\left(\nu, \beta, \mu, k^{\prime}\right)} \leq & C\left(\epsilon_{0}\right) C_{1} C_{2}\left(\sum_{\substack{1 \leq \ell_{1} \leq D_{1}-1 \\
1 \leq \ell_{2} \leq D_{2}-1}} \mathcal{C}_{\ell_{1} \ell_{2}}\right) \\
& \times\left\|\omega_{1}(\tau, m)-\omega_{2}(\tau, m)\right\|_{\left(\nu, \beta, \mu, k^{\prime}\right)} .
\end{aligned}
$$

Let $\epsilon_{0}>0$ be such that $C\left(\epsilon_{0}\right) C_{1} C_{2}\left(\sum_{1 \leq \ell_{1} \leq D_{1}-1,1 \leq \ell_{2} \leq D_{2}-1} \mathcal{C}_{\ell_{1} \ell_{2}}\right) \leq 1 / 2$. This entails that $\mathcal{H}_{\epsilon}$ is a contractive map in $\bar{B}(0, \varpi) \subseteq \operatorname{Exp}_{\left(v, \beta, \mu, k^{\prime}\right)}^{d}$.

As a consequence of Lemma 3, we achieve the next result.

Proposition 4 Assume that the hypotheses of Lemma 3 hold. Let $\varpi>0$. Then there exist $\epsilon_{0}>0$ and $\xi_{\psi}>0$ such that if $C_{\psi}<\xi_{\psi}$, then for every $\epsilon \in D\left(0, \epsilon_{0}\right)$, equation (21) admits a solution $\omega(\tau, m, \epsilon) \in \operatorname{Exp}_{\left(v, \beta, \mu, k^{\prime}\right)}^{d}$, with $\|\omega(\tau, m, \epsilon)\|_{\left(v, \beta, \mu, k^{\prime}\right)} \leq \varpi$.

Proof By the classical fixed point theorem in Banach spaces, Lemma 3 guarantees the existence of a function $\omega(\tau, m, \epsilon) \in B(0, \varpi) \subseteq \operatorname{Exp}_{\left(\nu, \beta, \mu, k^{\prime}\right)}^{d}$ such that $\mathcal{H}_{\epsilon}(\omega(\tau, m, \epsilon))=\omega(\tau, m, \epsilon)$ for every $\epsilon \in D\left(0, \epsilon_{0}\right)$. Moreover, the function $\omega(\tau, m, \epsilon)$ depends holomorphically on $\epsilon \in D\left(0, \epsilon_{0}\right)$. One can directly check that this fixed point is a solution of problem (21). 


\section{Actual solutions of auxiliary problem (21)}

Let $\mathcal{E}$ be a bounded sector of $\mathbb{C}^{\star}$ with vertex at the origin. Let $d \in \mathbb{R}$ be the bisecting direction of an unbounded sector $S_{d}$ satisfying the hypotheses of Proposition 4 . Let $\omega_{d}$ be the solution of (21) constructed in Proposition 4. Let $\tilde{\mathcal{T}}_{1}$ be a bounded sector with vertex at the origin, i.e., $\tilde{\mathcal{T}}_{1}=\{z \in \mathbb{C}: \arg (z) \in(\alpha, \beta),|z|<\rho\}$ for some $\alpha<\beta$ and $\rho>0$, and let $\tilde{\mathcal{T}}_{2}$ be an unbounded sector with vertex at the origin. We choose each of the previous sectors and small enough $\delta>0$ in order to satisfy that

$$
k_{j} \xi-k_{j} \arg \left(T_{j}\right) \in\left(-\frac{\pi}{2}+\delta, \frac{\pi}{2}-\delta\right), \quad j=1,2,
$$

for all $T_{j} \in \tilde{\mathcal{T}}_{j}$, some $\xi \in \mathbb{R}$ (which might depend on $T_{1}$ and $T_{2}$ ) under the assumption that $e^{\xi \sqrt{-1}} \in S_{d}$.

In particular, observe that there exists $\delta_{1}>0$ such that $\cos \left(k_{1} \xi-k_{1} \arg \left(T_{1}\right)\right)>\delta_{1}$ for every $T_{1} \in \tilde{\mathcal{T}}_{1}$. Moreover, there exists $\delta_{2}>0$ such that $\cos \left(k_{2} \xi-k_{2} \arg \left(T_{2}\right)\right)>\delta_{2}$ for every $T_{2} \in \tilde{\mathcal{T}}_{2}$.

The function $U_{\xi}$ defined in (18) turns out to be an actual solution of auxiliary problem (21) in the domain $\tilde{\mathcal{T}}_{1} \times \tilde{\mathcal{T}}_{2} \times \mathbb{R} \times \mathcal{E}$. Moreover, the following estimates hold:

$$
\begin{aligned}
& \left|U_{\xi}\left(T_{1}, T_{2}, m, \epsilon\right)\right| \\
& \leq \frac{\varpi(1+|m|)^{-\mu}}{e^{\beta|m|}} \int_{0}^{\infty} e^{\nu r^{k^{\prime}}} \\
& \quad \times \exp \left(-\frac{r^{k_{1}}}{\left|T_{1}\right|^{k_{1}}} \cos \left(k_{1} \xi-k_{1} \arg \left(T_{1}\right)\right)-\frac{r^{k_{2}}}{\left|T_{2}\right|^{k_{2}}} \cos \left(k_{2} \xi-k_{2} \arg \left(T_{2}\right)\right)\right) d r \\
& \leq \varpi(1+|m|)^{-\mu} e^{-\beta|m|} L\left(\left|T_{1}\right|,\left|T_{2}\right|\right),
\end{aligned}
$$

where

$$
L\left(\left|T_{1}\right|,\left|T_{2}\right|\right)=\int_{0}^{\infty} e^{\nu r^{k^{\prime}}} \exp \left(-\frac{r^{k_{1}}}{\left|T_{1}\right|^{k_{1}}} \delta_{1}-\frac{r^{k_{2}}}{\left|T_{2}\right|^{k_{2}}} \delta_{2}\right) d r
$$

Due to $k^{\prime} \in\left(k_{1}, k_{2}\right)$, the function $L(x, y)$ is well defined in $\left\{(x, y) \in \mathbb{R}^{2}: x \geq 0, y \geq 0\right\}$.

We write $L\left(\left|T_{1}\right|,\left|T_{2}\right|\right)=L_{1}\left(\left|T_{1}\right|,\left|T_{2}\right|\right)+L_{2}\left(\left|T_{1}\right|,\left|T_{2}\right|\right)$, with

$$
\begin{aligned}
& L_{1}\left(\left|T_{1}\right|,\left|T_{2}\right|\right)=\int_{0}^{\rho} e^{\nu r^{k^{\prime}}} e^{-\frac{r^{k_{1}}}{\left|T_{1}\right|^{k_{1}}} \delta_{1}-\frac{r^{k_{2}}}{\left|T_{2}\right|^{k_{2}}} \delta_{2}} d r, \\
& L_{2}\left(\left|T_{1}\right|,\left|T_{2}\right|\right)=\int_{\rho}^{\infty} e^{\nu r^{k^{\prime}}} e^{-\frac{r^{k_{1}}}{\left|T_{1}\right|^{k_{1}}} \delta_{1}-\frac{r^{k_{2}}}{\left|T_{2}\right|^{k_{2}}} \delta_{2}} d r
\end{aligned}
$$

for some $\rho>0$.

The proof of the following technical lemma is left to Sect. 4 at the end of the work in order not to interfere with the ongoing arguments.

Lemma 4 The following statements hold:

(1) There exists $C_{1}>0$ such that $0<L_{1}\left(\left|T_{1}\right|,\left|T_{2}\right|\right) \leq C_{1}$ for all $\left|T_{1}\right|,\left|T_{2}\right| \geq 0$. 
(2.a) Let $\rho_{1}>0$. There exists large enough $\rho_{2}^{\infty}>0$ such that

$$
\begin{aligned}
L_{2}\left(\left|T_{1}\right|,\left|T_{2}\right|\right) \leq & C_{2 a} e^{-\frac{\rho^{k_{1}}}{\left|T_{1}\right|^{k_{1}}} \delta_{1}}\left|T_{2}\right|^{\frac{k_{2}}{k_{2}-k^{\prime}}} \\
& \times \exp \left(v^{\left(1-k^{\prime} / k_{2}\right)^{-1}}\left(\frac{1}{\delta_{2}}\right)^{k^{\prime} /\left(k_{2}-k^{\prime}\right)}\left|T_{2}\right|^{k_{2} k^{\prime} /\left(k_{2}-k^{\prime}\right)}\right)
\end{aligned}
$$

for all $\left|T_{1}\right|<\rho_{1}$ and $\left|T_{2}\right|>\rho_{2}^{\infty}$, and some $C_{2 a}>0$.

(2.b) Let $\rho_{1}, \rho_{2}>0$. Then it holds that

$$
L_{2}\left(\left|T_{1}\right|,\left|T_{2}\right|\right) \leq C_{2 b} \exp \left(-\frac{\rho^{k_{1}}}{\left|T_{1}\right|^{k_{1}}} \delta_{1}\right) \exp \left(-\frac{\rho^{k_{2}}}{2\left|T_{2}\right|^{k_{2}}} \delta_{2}\right)
$$

for all $\left|T_{1}\right|<\rho_{1}$ and $\left|T_{2}\right|<\rho_{2}$, and some $C_{2 b}>0$.

Following our new approach, we are able to construct global solutions $U_{\xi}\left(T_{1}, T_{2}, m, \epsilon\right)$ in the time variable $T_{2}$ on an unbounded sector $\tilde{\mathcal{T}}_{2}$. This was not possible in our two previous studies $[15,18]$, where only local in time solutions were built up. This feature allows us to study the asymptotic expansions w.r.t. $\epsilon$ in two different situations: when $T_{2}$ remains in a prescribed bounded domain (which is related to the forthcoming outer solution, constructed for the main equation (10)) and when $T_{2}$ tends to $\infty$ in a related manner with $\epsilon$ (linked to the inner solutions of (10) that we plan to build in Sect. 4.1).

\section{Inner and outer solutions of the main problem}

In this section, we preserve the conditions established in the statement of the main problem under study in Sect. 2. More precisely, we assume that conditions (6)-(9) hold on the elements involved in problem (10), with forcing term $f$ determined by the construction and conditions in (19)-(20).

Our main aim is to construct solutions of the main problem (10) together with their asymptotic behavior in different situations. This will be done via (14) and shape (18), as stated in our second approach in Sect. 2.2.

We first specify some geometric constructions on the domain of definition of the solutions. First, we recall the definition of a good covering in $\mathbb{C}^{\star}$, and that of a good covering of prescribed opening.

Definition 3 Let $\iota_{1}, \iota_{2} \geq 2$ be integers. We consider two sets $\left(\mathcal{E}_{h_{1}}^{0}\right)_{0 \leq h_{1} \leq \iota_{1}-1}$ and $\left(\mathcal{E}_{h_{2}}^{\infty}\right)_{0 \leq h_{2} \leq l_{2}-1}$, where $\mathcal{E}_{h_{1}}^{0}, \mathcal{E}_{h_{2}}^{\infty} \subseteq D\left(0, \epsilon_{0}\right)$ are open sectors with vertex at the origin which satisfy the following assumptions:

(i) $\mathcal{E}_{h_{1}}^{0} \cap \mathcal{E}_{h_{1}+1}^{0} \neq \emptyset$ for all $0 \leq h_{1} \leq \iota_{1}-1$ (with $\mathcal{E}_{\iota_{1}}^{0}:=\mathcal{E}_{0}^{0}$ ) and $\mathcal{E}_{h_{2}}^{\infty} \cap \mathcal{E}_{h_{2}+1}^{\infty} \neq \emptyset$ for all $0 \leq h_{2} \leq \iota_{2}-1$ (with $\mathcal{E}_{\iota_{2}}^{\infty}:=\mathcal{E}_{0}^{\infty}$ ).

(ii) The intersection of three different elements of each family is empty.

(iii) The union of the elements of each family covers a punctured disc centered at 0 in $\mathbb{C}$.

(iv) The opening of $\mathcal{E}_{h_{1}}^{0}$ is larger than $\pi /\left(\lambda_{2} k_{2}\right)$ for all $0 \leq h_{1} \leq \iota_{1}-1$.

Then we say that $\left(\mathcal{E}_{h_{1}}^{0}\right)_{0 \leq h_{1} \leq \iota_{1}-1}$ is a good covering of $\mathbb{C}^{\star}$ of opening $\pi /\left(\lambda_{2} k_{2}\right)$, and $\left(\mathcal{E}_{h_{2}}^{\infty}\right)_{0 \leq h_{2} \leq l_{2}-1}$ is just called a good covering in $\mathbb{C}^{\star}$.

Definition 4 Let $\mathcal{T}_{1}$ be a bounded sector with vertex at the origin, and let $\mathcal{T}_{2}$ be an unbounded sector with vertex at the origin. Let $\left(\mathcal{E}_{h_{1}}^{0}\right)_{0 \leq h_{1} \leq \iota_{1}-1}$ be a good covering of $\mathbb{C}^{\star}$ of 
opening $\pi /\left(\lambda_{2} k_{2}\right)$, and let $\left(\mathcal{E}_{h_{2}}^{\infty}\right)_{0 \leq h_{2} \leq l_{2}-1}$ be a good covering in $\mathbb{C}^{\star}$. For every $0 \leq h_{1} \leq$ $\iota_{1}-1$, let $S_{d_{h_{1}}}^{0}$ be an unbounded sector of bisecting direction $d_{h_{1}}$, and let $S_{d_{h_{2}}}^{\infty}$ be an unbounded sector of bisecting direction $d_{h_{2}}$ for all $0 \leq h_{2} \leq \iota_{2}-1$. We say that the set $\left\{\mathcal{T}_{1}, \mathcal{T}_{2},\left(\mathcal{E}_{h_{1}}^{0}\right)_{0 \leq h_{1} \leq \iota_{1}-1},\left(S_{h_{1}}^{0}\right)_{0 \leq h_{1} \leq \iota_{1}-1}\right\}$ is admissible if there exists $\delta>0$ such that

$$
k_{j} \xi_{h_{1}}-k_{j} \arg \left(\epsilon^{\lambda_{j}} t_{j}\right) \in\left(-\frac{\pi}{2}+\delta, \frac{\pi}{2}-\delta\right), \quad j=1,2
$$

for all $t_{j} \in \mathcal{T}_{j}$, some $\xi_{h_{1}} \in \mathbb{R}$ (which might depend on $t_{j}$ and $\epsilon$ ) such that $e^{\xi_{h_{1}} \sqrt{-1}} \in S_{d_{h_{1}}}$, and all $\epsilon \in \mathcal{E}_{h_{1}}^{0}$.

The set $\left\{\mathcal{T}_{1}, \mathcal{T}_{2},\left(\mathcal{E}_{h_{2}}^{\infty}\right)_{0 \leq h_{2} \leq l_{2}-1},\left(S_{h_{2}}^{0}\right)_{0 \leq h_{2} \leq \iota_{2}-1}\right\}$ is said to be admissible if it holds that there exists small enough $\delta>0$ with

$$
k_{j} \xi_{h_{2}}-k_{j} \arg \left(\epsilon^{\lambda_{j}} t_{j}\right) \in\left(-\frac{\pi}{2}+\delta, \frac{\pi}{2}-\delta\right), \quad j=1,2
$$

for all $t_{j} \in \mathcal{T}_{j}$, some $\xi_{h_{2}} \in \mathbb{R}$ (which might depend on $t_{j}$ and $\epsilon$ ) such that $e^{\xi_{h_{2}} \sqrt{-1}} \in S_{d_{h_{2}}}$, and all $\epsilon \in \mathcal{E}_{h_{2}}^{\infty}$.

\subsection{Construction and results on the inner solutions}

Definition 5 Let $\mu_{2}>\lambda_{2}$ be an integer in such a way that

$$
\lambda_{1} k_{1}>\left(\mu_{2}-\lambda_{2}\right)\left(\frac{1}{k^{\prime}}-\frac{1}{k_{2}}\right)^{-1} .
$$

Let $\chi_{2}^{\infty}$ be the bounded domain

$$
\chi_{2}^{\infty}=\left\{x_{2} \in \mathbb{C}^{\star}: r_{2, \infty}<\left|x_{2}\right|<R_{2, \infty}, \arg \left(x_{2}\right) \in\left(\alpha_{2, \infty}, \beta_{2, \infty}\right)\right\}
$$

for some real numbers $0<r_{2, \infty}<R_{2, \infty}$ and $\alpha_{2, \infty}<\beta_{2, \infty}$.

We assume that the good covering $\left(\mathcal{E}_{h_{2}}^{\infty}\right)_{0 \leq h_{2} \leq l_{2}-1}$ satisfies the next additional condition: for all $0 \leq h_{2} \leq \iota_{2}-1$, we can choose $\theta_{h_{2}} \in \mathbb{R}$ (which depends on $\mathcal{E}_{h_{2}}^{\infty}$ ) such that, for all $x_{2} \in \chi_{2}^{\infty}$ and $\epsilon \in \mathcal{E}_{h_{2}}^{\infty}$, the complex number

$$
t_{2}=\frac{x_{2}}{\epsilon^{\mu_{2}}} e^{\theta_{h_{2}} \sqrt{-1}}
$$

belongs to $\mathcal{T}_{2}$ for all $\epsilon \in \mathcal{E}_{h_{2}}^{\infty}$. Then we define the set

$$
\mathcal{T}_{2, \epsilon, \mu_{2}}:=\left\{\frac{x_{2}}{\epsilon^{\mu_{2}}} e^{\theta_{h_{2}} \sqrt{-1}}: x_{2} \in \chi_{2}^{\infty}\right\}
$$

Remark Observe that $\mathcal{T}_{2, \epsilon, \mu_{2}} \subseteq \mathcal{T}_{2}$ for every $\epsilon \in \mathcal{E}_{h_{2}}^{\infty}$ and $0 \leq h_{2} \leq \iota_{2}-1$. In addition to this, observe that $\mathcal{T}_{2, \epsilon, \mu_{2}}$ is a bounded domain for every $\epsilon \in \mathcal{E}_{h_{2}}^{\infty}$ for $0 \leq h_{2} \leq \iota_{2}-1$ with

$$
\lim _{\epsilon \rightarrow 0, \epsilon \in \mathcal{E}_{h_{2}}^{\infty}} \operatorname{dist}\left(\mathcal{T}_{2, \epsilon, \mu_{2}}, 0\right)=\infty
$$


Theorem 1 Let $\left\{\mathcal{T}_{1}, \mathcal{T}_{2},\left(\mathcal{E}_{h_{2}}^{\infty}\right)_{0 \leq h_{2} \leq \iota_{2}-1},\left(S_{h_{2}}^{\infty}\right)_{0 \leq h_{2} \leq \iota_{2}-1}\right\}$ be an admissible set. For every $0 \leq$ $h_{2} \leq \iota_{2}-1$ and $\epsilon \in \mathcal{E}_{h_{2}}^{\infty}$, the function

$$
\left(t_{1}, t_{2}, z\right) \mapsto u_{d_{h_{2}}}\left(t_{1}, t_{2}, z, \epsilon\right),
$$

where

$$
\begin{aligned}
u_{d_{h_{2}}}\left(t_{1}, t_{2}, z, \epsilon\right):= & \mathcal{F}^{-1}\left(m \mapsto U_{\xi_{h_{2}}}\left(\epsilon^{\lambda_{1}} t_{1}, \epsilon^{\lambda_{2}} t_{2}, m, \epsilon\right)\right)(z), \\
= & \frac{1}{(2 \pi)^{1 / 2}} \int_{-\infty}^{\infty} \int_{L_{\xi_{h_{2}}}} \omega_{d_{h_{2}}}(u, m, \epsilon) \exp \left(-\left(\frac{u}{\epsilon^{\lambda_{1}} t_{1}}\right)^{k_{1}}-\left(\frac{u}{\epsilon^{\lambda_{2}} t_{2}}\right)^{k_{2}}\right) \\
& \times \exp (i z m) \frac{d u}{u} d m,
\end{aligned}
$$

where $\omega_{d_{h_{2}}}(u, m, \epsilon)$ is constructed in Sect. 2.4, defines a bounded holomorphic function on $\mathcal{T}_{1} \times \mathcal{T}_{2, \epsilon, \mu_{2}} \times H_{\beta^{\prime}}$ for all $0<\beta^{\prime}<\beta$, which is an actual solution of (10), called an inner solution. Moreover, for every $\epsilon \in \mathcal{E}_{h_{2}}^{\infty} \cap \mathcal{E}_{h_{2}+1}^{\infty}$, there exist $\tilde{C}, \tilde{D}>0$ such that

$$
\begin{aligned}
& \sup _{t_{1} \in \mathcal{T}_{1}, x_{2} \in \chi_{2}^{\infty}, z \in H_{\beta^{\prime}}}\left|u_{d_{h_{2}+1}}\left(t_{1}, x_{2} \epsilon^{-\mu_{2}} e^{\theta_{h_{2}} \sqrt{-1}}, z, \epsilon\right)-u_{d_{h_{2}}}\left(t_{1}, x_{2} \epsilon^{-\mu_{2}} e^{\theta_{h_{2}} \sqrt{-1}}, z, \epsilon\right)\right| \\
& \quad \leq \tilde{C} \exp \left(-\frac{\tilde{D}}{|\epsilon|^{\lambda_{1} k_{1}}}\right) .
\end{aligned}
$$

Proof Let $0 \leq h_{2} \leq \iota_{2}-1$, and $d_{h_{2}}$ be the value $d$ determined in Proposition 4. The function $\omega(\tau, m, \epsilon) \in \operatorname{Exp}_{\left(v, \beta, \mu, k^{\prime}\right)}^{d_{h_{2}}}$ solves equation (21). This entails that $U_{\xi_{h_{2}}}\left(\epsilon^{\lambda_{1}} t_{1}, \epsilon^{\lambda_{2}} t_{2}, m, \epsilon\right)$ is a solution of (14), and its inverse Fourier transform with respect to $m$ turns out to be a solution of (10). The geometric construction of the domains involved guarantees that the map $\left(t_{1}, t_{2}, z\right) \mapsto u_{d_{h_{2}}}\left(t_{1}, t_{2}, z, \epsilon\right)$ represents a bounded holomorphic function defined in $\mathcal{T}_{1} \times \mathcal{T}_{2, \epsilon, \mu_{2}} \times H_{\beta^{\prime}}$ for every $0<\beta^{\prime}<\beta$, where $\epsilon$ belongs to $\mathcal{E}_{h_{2}}^{\infty}$.

Regarding the definition of $\tau \mapsto P_{m}(\tau)$ in (23) and (26), we get that all the roots of $P_{m}(\tau)$ are at positive distance, say $\rho>0$, to the origin. This entails that the integration path defining the difference of two consecutive inner solutions can be deformed as follows.

Let $0 \leq h_{2} \leq \iota_{2}-1$, fix $\in \in \mathcal{E}_{h_{2}}^{\infty} \cap \mathcal{E}_{h_{2}+1}^{\infty}$, and write $t_{2}=x_{2} \epsilon^{-\mu_{2}} e^{\theta_{h_{2}} \sqrt{-1}} \in \mathcal{T}_{2, \epsilon, \mu_{2}}$ for some $x_{2} \in \chi_{2}^{\infty}$ and $\theta_{h_{2}} \in \mathbb{R}$, as described in Definition 5. For every $t_{1} \in \mathcal{T}_{1}, z \in H_{\beta^{\prime}}$, we get that

$$
\begin{aligned}
u_{d_{h_{2}+1}}\left(t_{1}, t_{2}, z, \epsilon\right)-u_{d_{h_{2}}}\left(t_{1}, t_{2}, z, \epsilon\right) \\
=\frac{1}{(2 \pi)^{1 / 2}} \int_{-\infty}^{\infty} \int_{L_{\xi_{h_{2}+1}}} \omega(u, m, \epsilon) \Omega\left(u, \epsilon^{\lambda_{1}} t_{1}, \epsilon^{\lambda_{2}} t_{2}\right) \frac{d u}{u} e^{i z m} d m \\
\quad-\frac{1}{(2 \pi)^{1 / 2}} \int_{-\infty}^{\infty} \int_{L_{\xi_{h_{2}}}} \omega(u, m, \epsilon) \Omega\left(u, \epsilon^{\lambda_{1}} t_{1}, \epsilon^{\lambda_{2}} t_{2}\right) \frac{d u}{u} e^{i z m} d m=E_{1}-E_{2}+E_{3},
\end{aligned}
$$

with $\Omega\left(u, T_{1}, T_{2}\right)$ defined in (18), and where

$$
\begin{aligned}
& E_{1}:=\frac{1}{(2 \pi)^{1 / 2}} \int_{-\infty}^{\infty} \int_{L_{\xi_{h_{2}+1}, \rho / 2}} \omega(u, m, \epsilon) \Omega\left(u, \epsilon^{\lambda_{1}} t_{1}, \epsilon^{\lambda_{2}} t_{2}\right) \frac{d u}{u} e^{i z m} d m, \\
& E_{2}:=\frac{1}{(2 \pi)^{1 / 2}} \int_{-\infty}^{\infty} \int_{L_{\xi_{h_{2}}, \rho / 2}} \omega(u, m, \epsilon) \Omega\left(u, \epsilon^{\lambda_{1}} t_{1}, \epsilon^{\lambda_{2}} t_{2}\right) \frac{d u}{u} e^{i z m} d m,
\end{aligned}
$$


and where $L_{\xi_{h_{2}+1}, \rho / 2}:=[\rho / 2,+\infty) e^{\xi_{h_{2}+1} \sqrt{-1}}, L_{\xi_{h_{2}}, \rho / 2}:=[\rho / 2,+\infty) e^{\xi_{h_{2}} \sqrt{-1}}$, and

$$
E_{3}:=\frac{1}{(2 \pi)^{1 / 2}} \int_{-\infty}^{\infty} \int_{C_{h_{2}, h_{2}+1, \rho / 2}} \omega(u, m, \epsilon) \Omega\left(u, \epsilon^{\lambda_{1}} t_{1}, \epsilon^{\lambda_{2}} t_{2}\right) \frac{d u}{u} e^{i z m} d m
$$

where $C_{h_{2}, h_{2}+1, \rho / 2}$ is the arc of circle departing from $(\rho / 2) e^{\xi_{h_{2}+1} \sqrt{-1}}$, ending at $(\rho / 2) e^{\xi_{h_{2}} \sqrt{-1}}$. Bearing in mind that $\mu_{2}>\lambda_{2}$, (38) and Lemma 4, one can apply (2.a) in Lemma 4 to arrive at $\left|E_{1}\right|$ is upper bounded by

$$
\begin{aligned}
& \int_{-\infty}^{\infty} \int_{\rho / 2}^{\infty} \frac{\varpi}{(1+|m|)^{\mu}} e^{-\beta|m|} e^{\nu r^{k^{\prime}}} \exp \left(-\left(\frac{r}{\left|\epsilon^{\lambda_{1}} t_{1}\right|}\right)^{k_{1}} \delta_{1}-\left(\frac{r}{\left|\epsilon^{\lambda_{2}} t_{2}\right|}\right)^{k_{2}} \delta_{2}\right) d r e^{|m| \operatorname{Im}(z)} d m \\
& \leq \tilde{C}_{1} \int_{\rho / 2}^{\infty} \exp \left(v r^{k^{\prime}}\right) \exp \left(-\left(\frac{r}{\left|\epsilon^{\lambda_{1}} t_{1}\right|}\right)^{k_{1}} \delta_{1}-\left(\frac{r}{\left|\epsilon^{\lambda_{2}} t_{2}\right|}\right)^{k_{2}} \delta_{2}\right) d r \\
& \leq \tilde{C}_{1} C_{2 a} \exp \left(-\frac{(\rho / 2)^{k_{1}} \delta_{1}}{\left|\epsilon^{\lambda_{1}} t_{1}\right|^{k_{1}}}\right)\left|\epsilon^{\lambda_{2}} t_{2}\right|^{1+\frac{k_{2}}{k_{2}-k^{\prime}}} \exp \left(v^{\left(1-k^{\prime} / k_{2}\right)^{-1}}\left(\frac{1}{\delta_{2}}\right)^{\frac{k^{\prime}}{k_{2}-k^{\prime}}}\left|\epsilon^{\lambda_{2}} t_{2}\right|^{\frac{k_{2} k^{\prime}}{k_{2}-k^{\prime}}}\right) \\
& \leq \tilde{C}_{1} C_{2 a} \exp \left(-\frac{(\rho / 2)^{k_{1}} \delta_{1}}{\left.\left|\epsilon^{\lambda_{1}}\right|^{k_{1}} C_{\mathcal{T}}^{k_{1}}\right)\left|\epsilon^{\lambda_{2}}\right|^{1+\frac{k_{2}}{k_{2}-k^{\prime}}} C_{\chi_{2}^{\infty}}^{\frac{k_{2} k^{\prime}}{k^{\prime}-k^{\prime}}}|\epsilon|^{-\mu_{2}\left(1+\frac{k_{2}}{k_{2}-k^{\prime}}\right)}}\right. \\
& \quad \times \exp \left(v^{\left(1-k^{\prime} / k_{2}\right)^{-1}}\left(\frac{1}{\delta_{2}}\right)^{\frac{k^{\prime}}{k_{2}-k^{\prime}}}\left|\epsilon^{\lambda_{2}}\right|^{\frac{k_{2} k^{\prime}}{k_{2}-k^{\prime}}} C_{\chi_{2}^{\infty}}^{1+\frac{k_{2}}{k_{2}-k^{\prime}}}|\epsilon|^{-\mu_{2} \frac{k_{2} k^{\prime}}{k_{2}-k^{\prime}}}\right)
\end{aligned}
$$

for some positive constant $\tilde{C}_{1}, C_{\mathcal{T}, 1}, C_{\chi_{2}^{\infty}}>0$. Taking into account (37), we derive that

$$
\left|E_{1}\right| \leq \tilde{C}_{2} \exp \left(-\frac{\tilde{D}_{2}}{|\epsilon|^{\lambda_{1} k_{1}}}\right)
$$

for some $\tilde{C}_{2}, \tilde{D}_{2}>0$.

An analogous upper bound can be associated with $\left|E_{2}\right|$. We finally consider $\left|E_{3}\right|$. Analogous estimates as for the previous yield

$$
\begin{aligned}
\left|E_{3}\right| \leq & \frac{1}{(2 \pi)^{1 / 2}} \int_{-\infty}^{\infty} \int_{\xi_{h_{2}}}^{\xi_{h_{2}+1}} \frac{\varpi}{(1+|m|)^{\mu}} e^{-\beta|m|} e^{\nu(\rho / 2)^{k^{\prime}}} \\
& \times \exp \left(-\frac{(\rho / 2)^{k_{1}} \delta_{1}}{|\epsilon|^{\lambda_{1} k_{1}} C_{\mathcal{T}, 1}}-\frac{(\rho / 2)^{k_{2}} \delta_{2}|\epsilon|^{\mu_{2} k_{2}}}{|\epsilon|^{\lambda_{2} k_{2}} C_{\chi_{2}}}\right) d r e^{|m| \operatorname{Im}(z)} d m \\
\leq & \tilde{C}_{3} \exp \left(-\frac{(\rho / 2)^{k_{1}} \delta_{1}}{|\epsilon|^{\lambda_{1} k_{1}} C_{\mathcal{T}, 1}}\right)
\end{aligned}
$$

for some $\tilde{C}_{3}, C_{\mathcal{T}, 1}, C_{\chi_{2}^{\infty}}>0$. This entails

$$
\left|E_{3}\right| \leq \tilde{C}_{4} \exp \left(-\frac{\tilde{D}_{4}}{|\epsilon|^{\lambda_{1} k_{1}}}\right)
$$

for some $\tilde{C}_{4}, \tilde{D}_{4}>0$. We conclude (40) from (42) and (43). 
We now fix a different good covering to provide the asymptotic behavior of the outer solutions. Let $\iota_{1} \geq 2$ be an integer. We fix a good covering in $\mathbb{C}^{\star},\left(\mathcal{E}_{h_{1}}^{0}\right)_{0 \leq h_{1} \leq \iota_{1}-1}$ of opening $\frac{\pi}{\lambda_{2} k_{2}}$. We observe that (35), (36) are satisfied for $j=2$ under the second assumption in (6).

\subsection{Construction and results on the outer solutions}

Theorem 2 Let $\rho_{2}>0$ and $0 \leq h_{1} \leq \iota_{1}-1$. Let

$$
\left\{\mathcal{T}_{1}, \mathcal{T}_{2},\left(\mathcal{E}_{h_{1}}^{0}\right)_{0 \leq h_{1} \leq \iota_{1}-1},\left(S_{h_{1}}^{0}\right)_{0 \leq h_{1} \leq \iota_{1}-1}\right\}
$$

be an admissible set. For every $0 \leq h_{1} \leq \iota_{1}-1$, the function $\left(t_{1}, t_{2}, z, \epsilon\right) \mapsto u_{d_{h_{1}}}\left(t_{1}, t_{2}, z, \epsilon\right)$, defined by

$$
\begin{aligned}
& \mathcal{F}^{-1}\left(m \mapsto U_{\xi_{h_{1}}}\left(\epsilon^{\lambda_{1}} t_{1}, \epsilon^{\lambda_{2}} t_{2}, m, \epsilon\right)\right)(z) \\
& =\frac{1}{(2 \pi)^{1 / 2}} \int_{-\infty}^{\infty} \int_{L_{\xi_{h_{1}}}} \omega(u, m, \epsilon) \exp \left(-\left(\frac{u}{\epsilon^{\lambda_{1}} t_{1}}\right)^{k_{1}}-\left(\frac{u}{\epsilon^{\lambda_{2}} t_{2}}\right)^{k_{2}}\right) \\
& \quad \times \exp (i z m) \frac{d u}{u} d m,
\end{aligned}
$$

defines a holomorphic and bounded function of $\mathcal{T}_{1} \times\left(\mathcal{T}_{2} \cap D\left(0, \rho_{2}\right)\right) \times H_{\beta^{\prime}} \times \mathcal{E}_{h_{1}}^{0}$, solving (10). This solution is called an outer solution of (10).

For every two consecutive outer solutions associated with (10), which are jointly defined in $\mathcal{T}_{1} \times\left(\mathcal{T}_{2} \cap D\left(0, \rho_{2}\right)\right) \times H_{\beta^{\prime}} \times\left(\mathcal{E}_{h_{1}}^{0} \cap \mathcal{E}_{h_{1}+1}^{0}\right)$, it holds that

$$
\begin{aligned}
& \sup _{t_{1} \in \mathcal{T}_{1}, t_{2} \in\left(\mathcal{T}_{2} \cap D\left(0, \rho_{2}\right)\right), z \in H_{\beta^{\prime}}}\left|u_{d_{h_{1}+1}}\left(t_{1}, t_{2}, z, \epsilon\right)-u_{d_{h_{1}}}\left(t_{1}, t_{2}, z, \epsilon\right)\right| \\
& \leq \hat{C} \exp \left(-\frac{\hat{D}}{|\epsilon|^{\lambda_{2} k_{2}}}\right), \epsilon \in \mathcal{E}_{h_{1}}^{0} \cap \mathcal{E}_{h_{1}+1}^{0},
\end{aligned}
$$

for two positive constants $\hat{C}, \hat{D}>0$.

Proof Let $0 \leq h_{1} \leq \iota_{1}-1$. We proceed as in the first part of the proof of Theorem 1 , to arrive at the splitting

$$
u_{d_{h_{1}+1}}\left(t_{1}, t_{2}, z, \epsilon\right)-u_{d_{h_{1}}}\left(t_{1}, t_{2}, z, \epsilon\right)=E_{1}-E_{2}+E_{3}
$$

for every $t_{1} \in \mathcal{T}_{1}, t_{2} \in \mathcal{T}_{2} \cap D\left(0, \rho_{2}\right), z \in H_{\beta^{\prime}}$, and $\epsilon \in \mathcal{E}_{h_{1}}^{0} \cap \mathcal{E}_{h_{1}+1}^{0}$. According to Lemma 4, statement (2.b), one has

$$
\begin{aligned}
\left|E_{1}\right| & \leq \hat{C}_{1} C_{2 b} \exp \left(-\frac{(\rho / 2)^{k_{1}}}{\left|\epsilon^{\lambda_{1}} t_{1}\right|^{k_{1}}} \delta_{1}\right) \exp \left(-\frac{(\rho / 2)^{k_{2}} \delta_{2}}{2\left|\epsilon^{\lambda_{2}} t_{2}\right|^{k_{2}}}\right) \\
& \leq \hat{C}_{1} C_{2 b} \exp \left(-\frac{(\rho / 2)^{k_{2}} \delta_{2}}{2 \rho_{2}^{k_{2}}} \frac{1}{|\epsilon|^{\lambda_{2} k_{2}}}\right)
\end{aligned}
$$

for some $\hat{C}_{1}>0$, valid for every $\epsilon \in \mathcal{E}_{h_{1}}^{0} \cap \mathcal{E}_{h_{1}+1}^{0}, t_{1} \in \mathcal{T}_{1}, t_{2} \in\left(\mathcal{T}_{2} \cap D\left(0, \rho_{2}\right)\right)$, and $z \in H_{\beta^{\prime}}$. Analogous bounds hold for $\left|E_{2}\right|$. Regarding $\left|E_{3}\right|$, one can follow the same bounds as for $\left|E_{1}\right|$. We conclude (45). 


\section{Parametric Gevrey asymptotic expansions of the solutions}

This section is devoted to the study of the asymptotic expansions associated with the outer and inner solutions of (10), with respect to the perturbation parameter. We make use of the cohomological criterion for $k$-summability of formal power series with coefficients in a Banach space (see [2], p. 121, or [10], Lemma XI-2-6), known as the Ramis-Sibuya theorem. We first recall the main definition of this summability theory.

\section{1 $k$-Summable formal power series and the Ramis-Sibuya theorem}

Let $\left(\mathbb{E},\|\cdot\|_{\mathbb{E}}\right)$ be a complex Banach space.

Definition 6 Let $k \geq 1$ be an integer. A formal power series

$$
\hat{f}(\epsilon)=\sum_{n=0}^{\infty} f_{n} \epsilon^{n} \in \mathbb{E} \llbracket \epsilon \rrbracket
$$

is said to be $k$-summable with respect to $\epsilon$ in the direction $d \in \mathbb{R}$ if there exists a bounded holomorphic function $f$ defined in a bounded sector $V_{d}$ of bisecting direction $d$ and opening larger than $\pi / k$ and values in $\mathbb{E}$ such that it admits $\hat{f}(\epsilon)$ as its Gevrey asymptotic expansion of order $1 / k$ on $V_{d}$, i.e., for every proper subsector $V_{1}$ of $V_{d}$, there exist $D, M>0$ with

$$
\left\|f(\epsilon)-\sum_{n=0}^{N-1} f_{n} \epsilon^{n}\right\|_{\mathbb{E}} \leq D M^{N} \Gamma\left(\frac{N}{k}+1\right)|\epsilon|^{N}
$$

for every $N \geq 1$ and $\epsilon \in V_{1}$.

Such a function is unique and it is called the $k$-sum of the formal power series. Furthermore, we can reconstruct the function $f$ by means of the classical Borel-Laplace procedure.

Theorem 3 (RS) Let $\left(\mathcal{E}_{h}\right)_{0 \leq h \leq l-1}$ be a good covering in $\mathbb{C}^{\star}$. For all $0 \leq h \leq \iota-1$, let $G_{h}$ : $\mathcal{E}_{h} \rightarrow \mathbb{E}$ be a holomorphic function, and define the cocycle $\Theta_{h}(\epsilon):=G_{h+1}(\epsilon)-G_{h}(\epsilon)$, which is a holomorphic function defined in $Z_{h}=\mathcal{E}_{h+1} \cap \mathcal{E}_{h}$ into $\mathbb{E}$. We assume:

(1) $G_{h}$ is a bounded function as $\epsilon \in \mathcal{E}_{h}$ tends to $0 \in \mathbb{C}$ for every $0 \leq h \leq \iota-1$.

(2) $\Theta_{h}(\epsilon)$ is an exponentially flat function of order $k$ in $Z_{h}$ for all $0 \leq h \leq \iota-1$, meaning that there exist $C_{h}, A_{h}>0$ with

$$
\left\|\Theta_{h}(\epsilon)\right\|_{\mathbb{E}} \leq C_{h} \exp \left(-\frac{A_{h}}{|\epsilon|^{k}}\right), \quad \epsilon \in Z_{h}
$$

for all $0 \leq h \leq \iota-1$.

Then, for all $0 \leq h \leq \iota-1$, the functions $G_{h}(\epsilon)$ have a common formal power series $\hat{G}(\epsilon) \epsilon$ $\mathbb{E} \llbracket \epsilon \rrbracket$ as Gevrey asymptotic expansion of order $1 / k$ on $\mathcal{E}_{h}$. Moreover, if the opening of one sector $\mathcal{E}_{h_{0}}$ is barely larger than $\pi / k$, then $G_{h_{0}}(\epsilon)$ is promoted as the $k$-sum of $\hat{G}(\epsilon)$ on $\mathcal{E}_{h_{0}}$.

\subsection{Parametric Gevrey asymptotic expansions of the inner and outer solutions of the main problem}

In this section, we display the main results of the present work, namely the asymptotic behavior of the inner and outer solutions of (10), constructed in the previous section. In 
the present section, we assume that conditions (6)-(9) hold on the elements involved in the main equation under study (10), with forcing term $f$ determined by the construction and conditions in (19)-(20). We depart from two good coverings $\left(\mathcal{E}_{h_{2}}^{\infty}\right)_{0 \leq h_{2} \leq l_{2}-1}$ of $\mathbb{C}^{\star}$ and $\left(\mathcal{E}_{h_{1}}^{0}\right)_{0 \leq h_{1} \leq \iota_{1}-1}$ of $\mathbb{C}^{\star}$, this second good covering with opening $\pi /\left(\lambda_{2} k_{2}\right)$; and choose $\mathcal{T}_{1}, \mathcal{T}_{2}$ satisfying (35), (36). We also fix $\chi_{2}^{\infty}$ as in Definition 5, which allows us to construct the family of inner solutions associated with the first good covering. In addition to this, we choose $\rho_{2}>0$ and the corresponding outer solutions associated with the second good covering.

Let $\mathbb{E}_{1}$ denote the Banach space of holomorphic and bounded functions defined in $\mathcal{T}_{1} \times$ $\chi_{2}^{\infty} \times H_{\beta^{\prime}}$ endowed with the sup. norm, and $\mathbb{E}_{2}$ the Banach space of holomorphic and bounded functions in $\mathcal{T}_{1} \times\left(\mathcal{T}_{2} \cap D\left(0, \rho_{2}\right)\right) \times H_{\beta^{\prime}}$, with sup. norm.

Theorem 4 The partial maps obtained from the inner solution of the main problem (10)

$$
\epsilon \mapsto u_{d_{h_{2}}}\left(t_{1}, \frac{x_{2}}{\epsilon^{\mu_{2}}} e^{\theta_{h_{2}} \sqrt{-1}}, z, \epsilon\right)
$$

have a common formal series $\hat{u}^{\infty}(\epsilon) \in \mathbb{E}_{1} \llbracket \epsilon \rrbracket$ as Gevrey asymptotic expansion of order $1 /\left(\lambda_{1} k_{1}\right)$ on $\mathcal{E}_{h_{2}}^{\infty}$ for $0 \leq h_{2} \leq \iota_{2}-1$.

Each of the partial maps obtained from the outer solution of the main problem (10), $\epsilon \mapsto$ $u_{d_{h_{1}}}\left(t_{1}, t_{2}, z, \epsilon\right)$ is the $\lambda_{2} k_{2}$-sum of a common formal power series $\hat{u}^{0}(\epsilon) \in \mathbb{E}_{2} \llbracket \epsilon \rrbracket$ on $\mathcal{E}_{h_{1}}^{0}$ for $0 \leq h_{1} \leq \iota_{1}-1$.

Proof A consequence of the construction of the inner solutions in Sect. 4.1 is that, for all $0 \leq h_{2} \leq \iota_{2}-1$, the function $\tilde{u}_{d_{h_{2}}}: \epsilon \mapsto u_{d_{h_{2}}}\left(t_{1}, \frac{x_{2}}{\epsilon^{\mu_{2}}} e^{\theta_{h_{2}} \sqrt{-1}}, z, \epsilon\right)$ is a holomorphic map on $\mathcal{E}_{h_{2}}^{\infty}$, with values in the Banach space $\mathbb{E}_{1}$ for all $0 \leq h_{2} \leq \iota_{2}-1$. Again, for every index, we consider the function $G_{h_{2}}:=\tilde{u}_{d_{h_{2}}}$ in the (RS) theorem. Due to Theorem 1, we have that

$$
\left\|G_{h_{2}+1}(\epsilon)-G_{h_{2}}(\epsilon)\right\|_{\mathbb{E}_{1}} \leq \tilde{C} \exp \left(-\frac{\tilde{D}}{|\epsilon|^{\lambda_{1} k_{1}}}\right)
$$

for every $\epsilon \in \mathcal{E}_{h_{2}}^{\infty} \cap \mathcal{E}_{h_{2}+1}^{\infty}$. We apply the (RS) theorem in order to achieve the existence of a common formal power series $\hat{u}^{\infty}(\epsilon) \in \mathbb{E}_{1} \llbracket \epsilon \rrbracket$ such that $\epsilon \mapsto u_{d_{h_{2}}}\left(t_{1}, \frac{x_{2}}{\epsilon^{\mu_{2}}} e^{\theta_{h_{2}} \sqrt{-1}}, z, \epsilon\right)$ admits $\hat{u}^{\infty}(\epsilon)$ as its Gevrey asymptotic expansion of order $1 /\left(\lambda_{1} k_{1}\right)$, on $\mathcal{E}_{h_{2}}^{\infty}$, for every $0 \leq$ $h_{2} \leq \iota_{2}-1$.

For the second part of the proof, we consider the functions $\breve{u}_{d_{h_{1}}}: \epsilon \mapsto u_{d_{h_{1}}}\left(t_{1}, t_{2}, z, \epsilon\right)$ for $0 \leq h_{1} \leq \iota_{1}-1$, which turn out to be a holomorphic map on $\mathcal{E}_{h_{1}}^{0}$, with values in the Banach space $\mathbb{E}_{2}$. We put $G_{h_{1}}:=\check{u}_{d_{h_{1}}}$ in the (RS) theorem. Due to Theorem 4 , we have that

$$
\left\|G_{h_{1}+1}(\epsilon)-G_{h_{1}}(\epsilon)\right\|_{\mathbb{E}_{1}} \leq \tilde{C} \exp \left(-\frac{\tilde{D}}{|\epsilon|^{\lambda_{2} k_{2}}}\right)
$$

for every $\epsilon \in \mathcal{E}_{h_{1}}^{0} \cap \mathcal{E}_{h_{1}+1}^{0}$. We apply the (RS) theorem in order to achieve the existence of a common formal power series $\hat{u}^{0}(\epsilon) \in \mathbb{E}_{2} \llbracket \epsilon \rrbracket$ such that $\epsilon \mapsto u_{d_{h_{1}}}\left(t_{1}, t_{2}, z, \epsilon\right)$ admits $\hat{u}^{0}(\epsilon)$ as its Gevrey asymptotic expansion of order $1 /\left(\lambda_{2} k_{2}\right)$, on $\mathcal{E}_{h_{1}}^{0}$, for every $0 \leq h_{1} \leq \iota_{1}-1$. Watson's lemma (see [1]) guarantees that this function is unique with this property, leading to a summability result. 


\section{Proof of Lemma 4}

It is straightforward to check statement (1) in Lemma 4, due to $k^{\prime}<k_{2}$.

We proceed to giving proof to (2.a): let $\rho_{2}^{\infty}, \rho_{1}>0$. One has that $L_{2}\left(\left|T_{1}\right|,\left|T_{2}\right|\right)$ is upper bounded by

$$
e^{-\frac{\rho^{k_{1}}}{\left|T_{1}\right|^{k_{1}}} \delta_{1}} \int_{\rho}^{\infty} e^{\nu r^{k^{\prime}}} e^{-\frac{r^{k_{2}}}{\left|T_{2}\right|^{k_{2}} \delta_{2}}} d r \leq e^{-\frac{\rho^{k_{1}}}{\left|T_{1}\right|^{k_{1}}} \delta_{1}} \int_{0}^{\infty} e^{\nu r^{k^{\prime}}} e^{-\frac{r^{k_{2}}}{\left|T_{2}\right|^{k_{2}}} \delta_{2}} d r
$$

Let

$$
\mathcal{L}(x)=\int_{0}^{\infty} e^{\nu r^{k^{\prime}}} e^{-\frac{r^{k_{2}}}{x}} d r
$$

We focus on determining upper bounds for $\mathcal{L}(x)$, as $x \rightarrow \infty$. Such bounds have already been observed in the proof of Proposition 4 of our recent contribution [16]. We provide a complete set of arguments for the sake of better readability.

By dominated convergence and the series representation of $\exp \left(v r^{k^{\prime}}\right)$, we derive that

$$
\mathcal{L}(x)=\int_{0}^{\infty} \sum_{n \geq 0} \frac{\left(v r^{k^{\prime}}\right)^{n}}{n !} e^{-\frac{k^{k_{2}}}{x}} d r=\sum_{n \geq 0} \frac{v^{n}}{n !} \int_{0}^{\infty}\left(r^{k^{\prime}}\right)^{n} e^{-\frac{r^{k} k_{2}}{x}} d r
$$

for all $x>0$. Let

$$
\mathcal{L}_{n}(x)=\int_{0}^{\infty}\left(r^{k^{\prime}}\right)^{n} e^{-\frac{k_{2}}{x}} d r
$$

The change of variable $r^{k_{2}} / x=\tilde{r}$ yields

$$
\mathcal{L}_{n}(x)=x^{\frac{k^{\prime}}{k_{2}} n} x^{\frac{1}{k_{2}}} \frac{1}{k_{2}} \int_{0}^{\infty}(\tilde{r})^{\frac{k^{\prime}}{k_{2}} n+\frac{1}{k_{2}}-1} e^{-\tilde{r}} d \tilde{r}=\frac{1}{k_{2}} x^{\frac{1}{k_{2}}} x^{\frac{k^{\prime}}{k_{2}} n} \Gamma\left(\frac{k^{\prime}}{k_{2}} n+\frac{1}{k_{2}}\right) .
$$

As a result,

$$
\mathcal{L}(x)=\frac{1}{k_{2}} x^{\frac{1}{k_{2}}} \sum_{n \geq 0} \frac{\left(v x^{\frac{k^{\prime}}{k_{2}}}\right)^{n}}{n !} \Gamma\left(\frac{k^{\prime}}{k_{2}} n+\frac{1}{k_{2}}\right)=\frac{1}{k_{2}} x^{\frac{1}{k_{2}}} \sum_{n \geq 0}\left(\nu x^{\frac{k^{\prime}}{k_{2}}}\right)^{n} \frac{\Gamma\left(\frac{k^{\prime}}{k_{2}} n+\frac{1}{k_{2}}\right)}{\Gamma(n+1)} .
$$

We recall (see Appendix B in [2]) the beta integral formula

$$
B(\alpha, \beta)=\int_{0}^{1}(1-t)^{\alpha-1} t^{\beta-1} d t=\frac{\Gamma(\alpha) \Gamma(\beta)}{\Gamma(\alpha+\beta)} \leq 1, \quad \alpha, \beta \geq 1 .
$$

Regrading the previous formula, we deduce the existence of a constant $C_{1}>0$ such that

$$
\Gamma\left(\frac{k^{\prime}}{k_{2}} n+\frac{1}{k_{2}}\right) \Gamma\left(\left(1-\frac{k^{\prime}}{k_{2}}\right) n+\left(1-\frac{1}{k_{2}}\right)\right) \leq C_{1} \Gamma(n+1)
$$

for $n \geq \max \left\{\frac{k_{2}-1}{k^{\prime}}, \frac{1}{k_{2}-k^{\prime}}\right\}$. This yields the existence of $\tilde{C}_{1}>0$ such that

$$
\mathcal{L}(x) \leq \tilde{C}_{1} x^{\frac{1}{k_{2}}} \sum_{n \geq 0} \frac{\left(v x^{\frac{k^{\prime}}{k_{2}}}\right)^{n}}{\Gamma\left(\left(1-\frac{k^{\prime}}{k_{2}}\right) n+\left(1-\frac{1}{k_{2}}\right)\right)} .
$$


We recall the following bounds on the generalized Mittag-Leffler function (Wiman function), see [8]:

$$
E_{\alpha, \beta}(z)=\sum_{n \geq 0} \frac{z^{n}}{\Gamma(\beta+\alpha n)}, \quad \alpha, \beta>0, \alpha \in(0,2) .
$$

There exists $C_{2}>0$ such that

$$
E_{\alpha, \beta}(z) \leq C_{2} z^{\frac{1-\beta}{\alpha}} e^{z^{\frac{1}{\alpha}}}, \quad z \geq 1 .
$$

Regarding (47), we guarantee the existence of $C_{3}>0$ with

$$
\mathcal{L}(x) \leq C_{3} x^{\frac{1}{k_{2}-k^{\prime}}} e^{\frac{1}{\nu-\frac{k^{\prime}}{k_{2}}}} x^{\frac{k^{\prime}}{k_{2}-k^{\prime}}}, \quad x \geq 1
$$

As a result, we derive the formula in statement (2.a).

We proceed to giving upper bounds on $L_{2}\left(\left|T_{1}\right|,\left|T_{2}\right|\right)$ in case (2.b). We assume that $\left|T_{1}\right|<$ $\rho_{1}$ for some $\rho_{1}>0$ and $\left|T_{2}\right|<\rho_{2}$ for some $\rho_{2}>0$.

We write $L_{2}\left(\left|T_{1}\right|,\left|T_{2}\right|\right)=\exp \left(-\frac{\rho^{k_{1}}}{\left|T_{1}\right|^{k_{1}}} \delta_{1}\right) L_{2.1}\left(\left|T_{2}\right|^{k_{2}}\right)$, where

$$
L_{2.1}\left(\left|T_{2}\right|^{k_{2}}\right)=\int_{\rho}^{\infty} e^{\nu r^{k^{\prime}}} e^{-\frac{r^{k_{2}}}{\left|T_{2}\right|^{k_{2}} \delta_{2}}} d r
$$

We have

$$
\begin{aligned}
& L_{2.1}\left(\left|T_{2}\right|^{k_{2}}\right) \\
& \quad=\int_{\rho}^{\infty} e^{\nu r^{k^{\prime}}} e^{-\frac{r^{k_{2}}}{2\left|T_{2}\right|^{k_{2}}} \delta_{2}} e^{-\frac{r^{k_{2}}}{2\left|T_{2}\right|^{k_{2}}} \delta_{2}} d r \\
& \quad \leq e^{-\frac{\rho^{k_{2}}}{2\left|T_{2}\right|^{k_{2}}} \delta_{2}} \int_{\rho}^{\infty} e^{\nu r^{k^{\prime}}} e^{-\frac{r^{k_{2}}}{2\left|T_{2}\right|^{k_{2}}} \delta_{2}} d r \leq e^{-\frac{\rho{ }^{k_{2}}}{2\left|T_{2}\right|^{k_{2}}} \delta_{2}} \int_{\rho}^{\infty} e^{\nu r^{k^{\prime}}} e^{-\frac{r^{k_{2}}}{2 \rho_{2}} \delta_{2}} d r,
\end{aligned}
$$

being the last integral upper bounded by a positive constant for $k_{2}>k^{\prime}$. In conclusion, there exists some $C_{2.1}>0$ such that the statement in (2.b) holds.

\section{Acknowledgements}

The authors express their gratitude to the anonymous referee for his valuable remark concerning the important fact, we mentioned in the introduction, that the special solution to our main problem (1) solves (4) under the compatibility condition (5). We thank also the referee for his accurate comments and suggestions which have helped to improve the quality of the paper.

\section{Funding}

The authors are partially supported by the research project MTM2016-77642-C2-1-P of Ministerio de Economía, Industria y Competitividad, Spain. The first author is partially supported by Comunidad de Madrid and Universidad de Alcalá under project CM-JIN-2019-010.

\section{Availability of data and materials}

Data sharing is not applicable to this article as no datasets were generated or analysed during the current study. 
Authors' contributions

All authors contributed equally and significantly in writing this paper and typed, read, and approved the final manuscript.

\section{Author details}

'Departamento de Física y Matemáticas, University of Alcalá, Alcalá de Henares, Spain. ²Laboratoire Paul Painlevé, University of Lille 1, Villeneuve d'Asca cedex, France.

\section{Publisher's Note}

Springer Nature remains neutral with regard to jurisdictional claims in published maps and institutional affiliations.

Received: 18 September 2019 Accepted: 6 January 2020 Published online: 09 January 2020

\section{References}

1. Balser, W.: From Divergent Power Series to Analytic Functions. Theory and Application of Multisummable Power Series. Lecture Notes in Mathematics, vol. 1582, x+108 pp. Springer, Berlin (1994)

2. Balser, W.: Formal Power Series and Linear Systems of Meromorphic Ordinary Differential Equations. Universitext, xviii+299 pp. Springer, New York (2000)

3. Bender, C., Orszag, S.: Advanced Mathematical Methods for Scientists and Engineers. I. Asymptotic Methods and Perturbation Theory, xiv+593 pp. Reprint of the 1978 original. Springer, New York (1999).

4. Canalis-Durand, M., Mozo-Fernández, J., Schäfke, R.: Monomial summability and doubly singular differential equations. J. Differ. Equ. 233(2), 485-511 (2007)

5. Carrillo, S.A., Mozo-Fernández, J.: Tauberian properties for monomial summability with applications to Pfaffian systems. J. Differ. Equ. 261(12), 7237-7255 (2016)

6. Carrillo, S.A., Mozo-Fernández, J.: An extension of Borel-Laplace methods and monomial summability. J. Math. Anal. Appl. 457(1), 461-477 (2018)

7. Eckhaus, W: Asymptotic Analysis of Singular Perturbations. Studies in Mathematics and Its Applications, vol. 9, xi+287 pp. North-Holland, Amsterdam (1979)

8. Erdelyi, A., Magnus, W., Oberhettinger, F., Tricomi, F.G.: Higher Transcendental Functions, vol. III. McGraw-Hill, New York (1955)

9. Fruchard, A., Schäfke, R.: Composite Asymptotic Expansions. Lecture Notes in Mathematics, vol. 2066, x+161 pp. Springer, Heidelberg (2013)

10. Hsieh, P., Sibuya, Y.: Basic Theory of Ordinary Differential Equations. Universitext. Springer, New York (1999)

11. Lagerstrom, P.: Matched Asymptotic Expansions. Ideas and Techniques. Applied Mathematical Sciences, vol. 76 , xii+250 pp. Springer, New York (1988)

12. Lastra, A., Malek, S.: On parametric Gevrey asymptotics for some nonlinear initial value Cauchy problems. J. Differ. Equ. 259(10), 5220-5270 (2015)

13. Lastra, A., Malek, S.: On parametric multisummable formal solutions to some nonlinear initial value Cauchy problems. Adv. Differ. Equ. 2015, 200 (2015)

14. Lastra, A., Malek, S.: Multi-level Gevrey solutions of singularly perturbed linear partial differential equations. Adv. Differ. Equ. 21(7-8), 767-800 (2016)

15. Lastra, A., Malek, S.: On parametric Gevrey asymptotics for some initial value problems in two asymmetric complex time variables. Results Math. 73(4), Art. 155 (2018)

16. Lastra, A., Malek, S.: On singularly perturbed linear initial value problems with mixed irregular and Fuchsian time singularities. J. Geom. Anal. (2019). https://doi.org/10.1007/s12220-019-00221-3

17. Lastra, A., Malek, S.: Multiscale Gevrey asymptotics in boundary layer expansions for some initial value problem with merging turning points. Adv. Differ. Equ. 24(1-2), 69-136 (2019)

18. Lastra, A., Malek, S.: On parametric Gevrey asymptotics for some nonlinear initial value problems in two complex time variables. Asymptot. Anal. (2020, to appear)

19. Mozo-Fernández, J., Schäfke, R.: Asymptotic expansions and summability with respect to an analytic germ. Publ. Math. 63(1), 3-79 (2019)

20. O'Malley, R.: Singular Perturbation Methods for Ordinary Differential Equations. Applied Mathematical Sciences, vol. 89, viii+225 pp. Springer, New York (1991)

21. Skinner, L.: Singular Perturbation Theory, $x+85$ pp. Springer, New York (2011)

22. Wasow, W.: Linear Turning Point Theory. Applied Mathematical Sciences, vol. 54, ix+246 pp. Springer, New York (1985)

23. Yamazawa, $H_{\text {., }}$ Yoshino, M.: Parametric Borel summability for some semilinear system of partial differential equations. Opusc. Math. 35(5), 825-845 (2015)

24. Yoshino, M.: Parametric Borel summability of partial differential equations of irregular singular type. In: Analytic, Algebraic and Geometric Aspects of Differential Equations. Trends Math., pp. 455-471. Springer, Cham (2017) 